\title{
LARGE DEVIATIONS UPPER BOUNDS AND CENTRAL LIMIT THEOREMS FOR NON-COMMUTATIVE FUNCTIONALS OF GAUSSIAN LARGE RANDOM MATRICES
}

\author{
Alice GUIONNET \\ UPMA, École Normale Supérieure de Lyon, 46 allée d'Italie, 69364 Lyon cedex 07, France
}

Received 5 June 2000, revised 6 March 2001

\begin{abstract}
We obtain large deviation upper bounds and central limit theorems for noncommutative functionals of large Gaussian band matrices and deterministic diagonal matrices with converging spectral measure. As a consequence, we derive such type of results for the spectral measure of Gaussian band matrices and Gaussian sample covariance matrices. ( $) 2002$ Éditions scientifiques et médicales Elsevier SAS
\end{abstract}

AMS classification: 60F10; 15A52; 60F05

Keywords: Large deviations; Random matrices; Central limit theorem

RÉSUMÉ. - Nous obtenons une borne supérieure de grandes déviations et un théorème central limite pour des fonctionelles non-commutatives de grandes matrices à bande gaussiennes aléatoires et d'une algèbre de matrices diagonales déterministes dont la mesure spectrale converge. Ceci nous permet de démontrer une borne supérieure de grandes déviations et un théorème central limite pour la mesure spectrale de matrices à bandes gaussiennes ainsi que des matrices de Wishart gaussiennes. @ 2002 Éditions scientifiques et médicales Elsevier SAS

\section{Introduction}

During the last decade, the understanding of the asymptotic behaviour of large random matrices has considerably improved since the pioneer works of Wigner [28], Arnold [1], Wachter [27], Wishart [29] and Pastur and Marchenko [20]. These papers were mainly motivated by Quantum Physics and proved convergence of the spectral measure of these matrices as their size goes to infinity under diverse assumptions on the distribution of their entries; Wigner [28] studied a random $N \times N$ Hermitian matrix with i.i.d. complex (or real) entries (except for the symmetry constraint), Wishart [29] (see also Wachter [27]) introduced the $N \times N$ Hermitian matrix $X_{N} X_{N}^{*}$ with $X_{N}$ a $N \times M$ matrix with i.i.d. complex (or real) entries, Pastur and Marchenko considered band matrices where the entries are non zero only on some band surrounding the origin and generalized 
sample covariance (or Wishart) matrices of the form $X_{N} R X_{N}^{*}$ with $X_{N}$ as above and a $M \times M$ deterministic matrix $R$ with converging spectral distribution (see [23,17,5]). We send the reader to [2] and [17] for reviews on the subject.

The fluctuations of the spectral measure around its limit for Wigner's matrix with Gaussian entries were first obtained by K. Johansson [18] (see also [9]). The fluctuations of the spectral measure around its expectation were studied under much more general assumptions over the entries and for most of the models described above (see $[22,8,16]$ and references therein). However, such statements are weaker than the result obtained by K. Johansson [18] for the Gaussian ensembles. In this paper, we shall generalize K. Johansson's type of results to band matrices and sample covariance matrices with Gaussian entries and for polynomial test functions.

Large deviations for the law of the spectral measure of Wigner's matrix with Gaussian entries were obtained in [3] and for related models in [4] and [13]. There is actually no clue how to extend these results to non-Gaussian entries. In [14], the authors obtained concentration inequalities for the spectral measure of the above matrices under various hypotheses on the distribution of the entries. However, even though this paper provides concentration on the right scale, there is no hope to deduce complementary lower bounds. Here, we shall obtain large deviation upper bounds for the deviations of the spectral measure of Gaussian band matrices, which we hope optimal. This result in turn provides a large deviation upper bound for the spectral measure of generalized Gaussian sample covariance matrices, but a full large deviation principle was very recently obtained in [15] by O. Zeitouni and myself in this restricted context. Observe at this point that the joint law of the eigenvalues of Gaussian band matrices (or Gaussian sample covariance matrices) is a priori complicated, being given by a $N \times N$ Jacobian which does not lead to simple formulae since the law of Gaussian band matrices are not invariant under the action of a group such as the unitary (or orthogonal) group on the contrary of Wigner's matrices. In particular, the techniques of [3] are useless here. In the direction of interests encountered in free probability, deviations of the non-commutative law of a couple of independent Gaussian Wigner's matrices were studied in [10] using a functional approach based on stochastic calculus. We shall follow a similar approach in this work.

However, the goal of this paper is not only to consider functions of the spectral measure of large random matrices but more general non-commutative functionals involving large random matrices and an algebra of deterministic diagonal matrices. Such functionals were already introduced in [23] where the author obtained law of large numbers type of statements for the normalized trace of these functionals thanks to free probability techniques (more precisely the notion of freeness with amalgamation). As a consequence, D. Shlyakhtenko deduced the convergence of the spectral measure for Gaussian band matrices. The strategy followed in this paper is intimately related to the ideas of [23] but we shall push forward the analysis to obtain large deviation upper bounds and central limit theorems. In particular, we define a good rate function governing the large deviations of these non-commutative functionals and a self adjoint positive definite operator defining the covariance of the central limit theorem. Large deviations results for non-commutative variables were already obtained in [10] and a central limit theorem in [9] for independent Gaussian Wigner's matrices. The main 
difference here is that we consider a single random matrix and a deterministic algebra of diagonal matrices. Some of our statements could be interpreted in terms of free probability. However, we shall not discuss this aspect in details here.

The paper is organized as follows; we begin with the introduction of our notations and results. We then introduce Itô's calculus for band matrices which is the key to all our proofs. In Section 4, we state and prove a large deviation upper bound. Studying the minimizer of our rate function, we deduce a law of large numbers theorem in Section 5. It is supplemented in Section 6 by a central limit theorem. We also describe in the next section how these results can be interpreted in terms of inhomogeneous sample covariance matrices.

Throughout this paper, we shall denote by, for two metric spaces $A$ and $B, \mathcal{C}^{p}(A, B)$ (resp. $\left.\mathcal{C}_{b}^{p}(A, B)\right)$ the set of (resp. bounded) $p$ times continuously differentiable functions from $A$ into $B$. When $A=B$, we denote in short $\mathcal{C}_{b}^{p}(A, A)=\mathcal{C}_{b}^{p}(A)$. When $p=0$, corresponding to continuous functions, we drop the subscript 0 to simplify the notations.

\section{Notations and statement of the results}

Hereafter, $\mathcal{M}_{N}$ will denote the set of $N \times N$ matrices with complex independent entries. $\mathcal{H}_{N}$ will be the subset of $\mathcal{M}_{N}$ of Hermitian matrices. We set $\mathcal{M}=\bigcup_{N \in \mathbb{N}} \mathcal{M}_{N}$ and $\mathcal{H}=\bigcup_{N \in \mathbb{N}} \mathcal{H}_{N}$. tr will denote the natural extension of the trace to $\mathcal{M}$ given, for any $A \in \mathcal{M}_{N}, N \in \mathbb{N}$, by $\operatorname{tr}(A)=\sum_{i=1}^{N} A_{i i}$ and $\operatorname{tr}_{N}$ the normalized $\operatorname{trace}^{\operatorname{tr}_{N}}(A)=N^{-1} \operatorname{tr}(A)$ for $A \in \mathcal{M}_{N}, N \in \mathbb{N}$. We shall consider, for $N \in \mathbb{N}$, the random matrix in $\mathcal{H}_{N}$

$$
\left(X_{N}\right)_{i j}=\left(H_{N}\right)_{i j} \psi_{N}(i, j)^{\frac{1}{2}},
$$

where $H_{N}$ is a Hermitian matrix with complex Gaussian entries with covariance $N^{-1}$ and $\psi_{N}$ is a non-negative symmetric function on $\{1, \ldots, N\}^{2}$ which can be decomposed as

$$
\psi_{N}(x, y)=\int \sigma_{\tau}^{N}(x) \sigma_{\tau}^{N}(y) \mathrm{d} p(\tau)
$$

with a measure $p$ on a Polish space $(\Omega, \Sigma)$ with finite mass, and bounded functions $\left(\sigma_{\tau}^{N}, \tau \in \Omega\right)$ on $\{1, \ldots, N\}$ such that $\tau \rightarrow \sigma_{\tau}^{N}(x)$ is measurable for the sigma-algebra $\Sigma$ for any $x \in\{1, \ldots, N\}$. We can assume without loss of generality that the total mass of $p$ is one to simplify the notations. We shall assume that, if $\Delta_{\tau}^{N}$ denotes the $N \times N$ matrix with diagonal elements $\left(\sigma_{\tau}^{N}(i), 1 \leqslant i \leqslant N\right)$.

(H0). - For any $\left(\tau_{1}, \ldots, \tau_{n}\right) \in \Omega^{n}, n \in \mathbb{N}$, the joint distribution (in the non-commutative sense) of $\left(\Delta_{\tau_{1}}, \ldots, \Delta_{\tau_{n}}\right)$ converges, i.e., there exists a probability measure $m_{\tau_{1}, \ldots, \tau_{n}}$ on $\mathbb{R}$ so that for every bounded continuous function $f$ on $\mathbb{R}$,

$$
\lim _{N \rightarrow \infty} \frac{1}{N} \sum_{i=1}^{N} f\left(\prod_{j=1}^{n} \sigma_{\tau_{j}}^{N}(i)\right)=\int f(x) \mathrm{d} m_{\tau_{1}, \ldots, \tau_{n}}(x) .
$$

Further, we suppose that $T \equiv \sup _{\tau \in \Omega} \sup _{N \in \mathbb{N}} \sup _{x \in\{1, \ldots, N\}}\left|\sigma_{\tau}^{N}(x)\right|<\infty$. 
Following [10], it is convenient to consider, in order to use the powerful tool of stochastic differential calculus, $X_{N}$ as the value at time one of the $\mathcal{H}_{N}$-valued process

$$
\left(X_{N}(t)\right)_{i j}=\left(H_{N}(t)\right)_{i j} \psi_{N}(i, j)^{\frac{1}{2}},
$$

where $H_{N}(t)$ is the Hermitian Brownian motion which is described on the space $\mathcal{H}_{N}$ of Hermitian matrices of dimension $N$ as the Markov process $\left(H_{N}(t)\right)_{t \in \mathbb{R}^{+}}$with values in $\mathcal{H}_{N}$ and independent complex Brownian motions entries so that

$$
E\left[H_{N}^{i, j}(t) H_{N}^{k, l}(s)\right]=\frac{t \wedge s}{N} \delta_{i}^{l} \delta_{k}^{j} .
$$

More precisely, we can construct the entries $\left\{H_{N}^{i, j}(t), t \geqslant 0,(i, j) \in\{1, \ldots, N\}\right\}$ via independent real valued Brownian motions $\left(\beta_{i, j}, \beta_{k, l}^{\prime}\right)_{\substack{1 \leqslant k<j \leqslant N \\ 1 \leqslant i \leqslant j}}$ by

$$
H_{N}^{k, l}=\frac{1}{\sqrt{\left(1+\delta_{k<l}\right) N}}\left(\beta_{k, l}+i \delta_{k<l} \beta_{k, l}^{\prime}\right) \quad \text { if } k \leqslant l .
$$

To take into account the inhomogeneity of the covariance of $X_{N}$, we shall, following D. Shlyakhtenko [23], consider jointly the matrix-valued process $\left(X_{N}(t), t \in[0,1]\right)$ and diagonal matrices. To this end, let us introduce a set $\mathbb{D}$ of sequences $\Delta$ of uniformly bounded converging diagonal matrices $\Delta^{N}$ of $\mathcal{H}_{N}$ (hence with real entries) that is sequences $\Delta=\left(\Delta^{N}\right)_{N \in \mathbb{N}}$ so that, if $\left(\lambda_{1}^{N}, \ldots, \lambda_{N}^{N}\right)$ denotes the eigenvalues of $\Delta^{N}$,

$$
\sup _{N \in \mathbb{N}} \sup _{i \in\{1, \ldots, N\}}\left|\lambda_{i}^{N}\right|<\infty
$$

and $\frac{1}{N} \sum_{i=1}^{N} \delta_{\lambda_{i}^{N}}$ converges as $N$ tends to infinity for the weak topology, i.e., there exists a probability measure $m_{\Delta}$ on $\mathbb{R}$ so that for any function $f \in \mathcal{C}_{b}(\mathbb{R})$,

$$
\lim _{N \rightarrow \infty} \frac{1}{N} \sum_{i=1}^{N} f\left(\lambda_{i}^{N}\right)=\int f(x) \mathrm{d} m_{\Delta}(x) .
$$

In the sequel, we write in short

$$
m(\Delta)=\int x \mathrm{~d} m_{\Delta}(x), \quad \forall \Delta \in \mathcal{D} .
$$

We shall consider a sub-algebra $\mathcal{D}$, that is stable by product and sum, of $\mathbb{D}$ containing the real vector space generated by the identity and the null matrices as well as the sequences

$$
\mathcal{D}_{\psi}=\left\{\Delta_{\tau}=\left(\Delta_{\tau}^{N}=\left(\delta_{i=j} \sigma_{\tau}^{N}(i)\right)_{1 \leqslant i, j \leqslant N}\right)_{N \in \mathbb{N}}, \tau \in \Omega\right\} .
$$

We endow $\mathbb{D}$ with the norm given, for any $\Delta, \tilde{\Delta} \in \mathbb{D}$ by

$$
|\Delta-\tilde{\Delta}|_{\infty}=\sup _{N \in \mathbb{N}} \sup _{i \in\{1, \ldots, N\}}\left|\Delta_{i}^{N}-\tilde{\Delta}_{i}^{N}\right|
$$


and assume that $\mathcal{D}$ is separable for this norm.

Examples (2.4). - (a) The first example one should keep in mind is when

$$
\sigma_{\tau}^{N}(i)=\sigma_{\tau}\left(\frac{i}{N}\right)
$$

with $\sigma_{\tau} \in \mathcal{C}_{b}([0,1], \mathbb{R})$ for $\tau \in \Omega$. In the sequel, we shall denote by $\Delta(\phi)$ the sequence

$$
\Delta(\phi) \equiv\left(\left(\Delta_{N}(\phi)\right)_{i j}=\delta_{i=j} \phi\left(\frac{i}{N}\right), i, j \in\{1, \ldots, N\}\right)_{N \in \mathbb{N}}
$$

for $\phi \in \mathcal{C}_{b}([0,1], \mathbb{R})$. One can choose $\mathcal{D}$ to be the set

$$
\mathcal{D}_{c} \equiv\left\{\Delta: \exists \phi \in \mathcal{C}_{b}([0,1], \mathbb{R}) ; \Delta=\Delta(\phi)\right\} .
$$

$\mathcal{D}_{c}$ is an algebra and is separable for ||$_{\infty}$ since $\mathcal{C}_{b}([0,1], \mathbb{R})$ is separable for the uniform norm. (2.3) is fulfilled with $m_{\Delta(\phi)}=\lambda_{[0,1]} \circ \phi^{-1}$ if $\lambda_{[0,1]}$ denotes the Lebesgue measure on $[0,1]$.

(b) However, the general scheme proposed above may be useful to include the case where, for instance,

$$
\sigma_{\tau}^{N}(i)=\sigma_{\tau}\left(\frac{i}{N}\right)+1_{0 \leqslant i \leqslant M_{N}} \tilde{\sigma}_{\tau}\left(\frac{i}{N}\right)
$$

for some positive real number $M_{N}$ and bounded continuous functions $\sigma_{\tau}$ and $\tilde{\sigma}_{\tau}$. We assume

$$
\lim _{N \rightarrow \infty} \frac{M_{N}}{N}=\alpha .
$$

Denoting, for $\phi, \tilde{\phi} \in \mathcal{C}_{b}([0,1], \mathbb{R}), \Delta(\phi, \tilde{\phi})$ the sequence

$$
\Delta^{N}(\phi, \tilde{\phi})_{i j}=\delta_{i=j}\left(\phi\left(\frac{i}{N}\right)+1_{0 \leqslant \frac{i}{N} \leqslant \frac{M_{N}}{N}} \tilde{\phi}\left(\frac{i}{N}\right)\right), \quad i, j \in\{1, \ldots, N\}, N \in \mathbb{N},
$$

we can choose $\mathcal{D}$ to be the separable algebra

$$
\mathcal{D}_{d}=\left\{\Delta: \exists \phi, \tilde{\phi} \in \mathcal{C}_{b}([0,1], \mathbb{R}) ; \Delta=\Delta(\phi, \tilde{\phi})\right\} .
$$

(2.3) is also easily checked with $m_{\Delta(\phi, \tilde{\phi})}=\lambda_{[0,1]} \circ \phi^{-1}+\lambda_{[0, \alpha]} \circ \tilde{\phi}^{-1}$ This second example will appear naturally when we shall consider generalized Wishart's matrices.

We shall see an element $\Delta$ of $\mathbb{D}$ as a function from $\mathcal{H}$ into $\mathcal{H}$ by setting for any $X \in \mathcal{H}_{N}, N \in \mathbb{N}, \Delta(X)=\Delta^{N}$.

In [23], D. Shlyakhtenko considered the random variables

$$
\left\{\operatorname{tr}_{N}\left(P\left(X_{N}(1), \Delta_{1}^{N}, \ldots, \Delta_{n}^{N}\right)\right), \Delta_{1}, \ldots, \Delta_{n} \in \mathcal{D}, n \in \mathbb{N}\right\}
$$

for non-commutative polynomial functions $P$ of $n+1$ variables, and proved their convergence as $N$ goes to infinity. Because the associated topology inherited for instance 
on the spectral measure of $X_{N}$ is not the weak topology, we shall, as in [10], consider other test functions than polynomials. Such test functions shall belong to the set $\mathcal{E}(\mathbb{C})$ of functions on $\mathcal{H}$ so that for any $N \in \mathbb{N}, F \in \mathcal{E}(\mathbb{C})$ maps $\mathcal{H}_{N}$ into $\mathcal{M}_{N} \cdot \mathcal{E}(\mathbb{R})$ will be the subset of Hermitian matrix-valued functions of $\mathcal{E}(\mathbb{C})$. Note that if $f$ is a real function, we can define the function $F$ on $\mathcal{H}$ so that, if $X \in \mathcal{H}, X=U^{*} D U$ for a diagonal matrix $D$ and a unitary matrix $U$,

$$
F(X)=U^{*} f(D) U, \quad f(D)_{i j}=\delta_{i=j} f\left(D_{i i}\right) .
$$

It is straightforward that $F$ belongs to $\mathcal{E}(\mathbb{R})$. In particular, for any $z \in \mathbb{C} \backslash \mathbb{R}, X \rightarrow$ $(z-X)^{-1}$ is an element of $\mathcal{E}(\mathbb{C})$. We shall be particularly interested in the following by the complex vector space $F_{\mathbb{C}}(X, \mathcal{D}) \subset \mathcal{E}(\mathbb{C})$ generated by

$$
\begin{aligned}
\{F: \mathcal{H} \rightarrow \mathcal{M} ; F(X)= & \prod_{1 \leqslant i \leqslant n}\left(z_{i}-\alpha_{i} X\right)^{-1} \Delta_{i}(X), \\
& \left.\left(z_{i}\right)_{1 \leqslant i \leqslant n} \in(\mathbb{C} \backslash \mathbb{R})^{n}, \alpha_{i} \in\{0,1\}, \Delta_{i} \in \mathcal{D}, n \in \mathbb{N}\right\} .
\end{aligned}
$$

Here, $\Pi \rightarrow$ denotes the non-commutative product. Observe that $F_{\mathbb{C}}(X, \mathcal{D})$ is an algebra since $\mathcal{D}$ is. Further, it contains 0 and 1 since $\mathcal{D}$ does. $F_{\mathbb{R}}(X, \mathcal{D})$ shall denote the real vector space of the Hermitian matrix-valued functions of $F_{\mathbb{C}}(X, \mathcal{D})$.

We shall prove the following law of large numbers

TheOREM (2.5). - Under $(\mathrm{H} 0)$, for any $F \in F_{\mathbb{C}}(X, \mathcal{D})$, any $t \in[0,1], \operatorname{tr}_{N}\left(F\left(X_{N}(t)\right)\right)$ converges almost surely as $N$ goes to infinity. Its limit, denoted by $\mu_{t}^{*}(F)$, is described in Section 5.

In particular, if $\psi_{N}$ is as in Examples (2.4) and taking functions of $F_{\mathbb{C}}(X, \mathcal{D})$ which are products of one Stieltjes functions and one diagonal matrix, we find that, if $\psi$ is the function on $[0,1]^{2}$ given by

$$
\psi(x, y)=\lim _{N \rightarrow \infty} \psi_{N}([N x],[N y]) .
$$

COROLlaRY (2.6). - Under (H0), for any $t \in[0,1]$, any $z$ in $\mathbb{C} \backslash \mathbb{R}$, any $\phi \in$ $\mathcal{C}_{b}([0,1], \mathbb{R})$,

$$
N^{-1} \sum_{i=1}^{N} \phi\left(\frac{i}{N}\right)\left[\left(z-X_{N}(t)\right)^{-1}\right]_{i i}
$$

converges almost surely towards $t^{-\frac{1}{2}} \int_{0}^{1} \phi(x) k\left(x, t^{-\frac{1}{2}} z\right) \mathrm{d} x$ where, if $K$ is the operator in $L^{2}([0,1])$ with kernel $\psi, k$ is the unique analytic solution of

$$
k(x, z)=(z-K(k(., z))(x))^{-1}
$$

so that $z k(x, z)$ goes to one as $|z|$ goes to infinity for any $x \in[0,1]$.

See Lemma (5.10) for details. 
Further, by density of $F_{\mathbb{C}}(X, \mathcal{D})$ in the set of non-commutative polynomial functions and controls of the normalized trace of moments of $X_{N}(t)$, we shall see that Theorem (2.5) implies that

COROLlaRY (2.7). - Under (H0), for any $t \in[0,1]$, any $\Delta_{1}, \Delta_{2}, \ldots, \Delta_{n} \in \mathcal{D}$, any non-commutative polynomial function $P$ of $n+1$ variables, $\operatorname{tr}_{N}\left(P\left(X_{N}(t), \Delta_{1}^{N}, \ldots, \Delta_{n}^{N}\right)\right)$ converges almost surely towards a well defined limit denoted by $\mu_{t}^{*}(P)$.

Hence, we find again the results of [23] and [20]. This last result is precised in Section 6 by a central limit theorem which validity requires the following extra hypotheses.

(H1). - For any $\Delta \in \mathcal{D}$,

$$
N\left(\operatorname{tr}_{N}\left(\Delta^{N}\right)-m(\Delta)\right)
$$

converges as $N$ goes to infinity towards a constant $c(\Delta)$.

Remark that, since $\mathcal{D}$ must contain $\mathcal{D}_{\psi}$, this last assumption also applies to $\left(\Delta_{\tau}, \tau \in\right.$ $\Omega)$. We shall also impose

(H2). - For any $\Delta_{1}, \ldots, \Delta_{n} \in \mathcal{D}$, any $m \in \mathbb{N}$, any non-commutative polynomial function $P$ of $n+m$ variables,

$$
\begin{aligned}
& \sup _{\tau_{1}, \ldots, \tau_{m} \in \Omega} \sup _{N \in \mathbb{N}} N \mid \operatorname{tr}_{N}\left(P\left(\Delta_{\tau_{1}}^{N}, \ldots, \Delta_{\tau_{m}}^{N}, \Delta_{1}^{N}, \ldots, \Delta_{n}^{N}\right)\right) \\
& \quad-m\left(P\left(\Delta_{\tau_{1}}, \ldots, \Delta_{\tau_{m}}, \Delta_{1}, \ldots, \Delta_{n}\right)\right) \mid<\infty .
\end{aligned}
$$

Then, we will show the

Theorem (2.8). - Under (H0), (H1) and (H2), for any $t \in[0,1]$, any $n \in \mathbb{N}$; any $\Delta_{1}, \ldots, \Delta_{n} \in \mathcal{D}$, any non-commutative polynomial function $P$ of $n+1$ variables $N\left(\operatorname{tr}_{N}\left(P\left(X_{N}(t), \Delta_{1}^{N}, \ldots, \Delta_{n}^{N}\right)\right)-\mu_{t}^{*}(P)\right)$ converges in law as $N$ goes to infinity towards a (eventually not centered) Gaussian law.

We send the reader to Section 6 for the definition of the mean and the covariance of the above Gaussian law. Let us give the following

Example (2.9). - We consider again the examples given in (2.4).

(a) In the first example, we consider the case where

$$
\sigma_{\tau}^{N}(i)=\sigma_{\tau}\left(\frac{i}{N}\right)
$$

To obtain a central limit theorem, we shall assume that $\sigma_{\tau}$ belongs to $\mathcal{C}_{b}^{1}([0,1], \mathbb{R})$ for $\tau \in \Omega$ and that, if $\|\quad\|_{u}$ is the uniform norm on $\mathcal{C}_{b}([0,1], \mathbb{R})$

$$
\sup _{\tau \in \Omega}\left\|\sigma_{\tau}^{\prime}\right\|_{u}<\infty .
$$

One can then choose $\mathcal{D}$ to be the set

$$
\mathcal{D}_{c}^{\prime}=\left\{\Delta(\phi), \phi \in \mathcal{C}_{b}^{1}([0,1], \mathbb{R})\right\} .
$$


$\mathcal{D}_{c}^{\prime}$ is clearly an algebra. Further, (H1) is fulfilled since for any $\phi \in \mathcal{C}_{b}^{1}([0,1])$,

$$
\lim _{N \rightarrow \infty} N\left(\frac{1}{N} \sum_{i=1}^{N} \phi\left(\frac{i}{N}\right)-\int_{0}^{1} \phi(x) \mathrm{d} x\right)=\frac{1}{2}(\phi(1)-\phi(0)):=c(\Delta(\phi)) .
$$

Also, observe that for any $\tau_{1}, \ldots, \tau_{m} \in \Omega$, any $\Delta_{1}, \ldots, \Delta_{n} \in \mathcal{D}$, any non-commutative polynomial function $P$ of $n+m$ variables, $P\left(\Delta_{\tau_{1}}^{N}, \ldots, \Delta_{\tau_{m}}^{N}, \Delta_{1}^{N}, \ldots, \Delta_{n}^{N}\right)=\Delta^{N}(\phi)$ for some $\phi \in \mathcal{C}_{b}^{1}([0,1], \mathbb{R})$ and that

$$
N\left|\operatorname{tr}_{N}\left(\Delta^{N}(\phi)\right)-\int_{0}^{1} \phi(x) \mathrm{d} x\right| \leqslant\left\|\phi^{\prime}\right\|_{u}
$$

shows that hypothesis $(\mathrm{H} 2)$ is easily derived from (2.10).

(b) In the case where

$$
\sigma_{\tau}^{N}(i)=\sigma_{\tau}\left(\frac{i}{N}\right)+1_{0 \leqslant i \leqslant M_{N}} \tilde{\sigma}_{\tau}\left(\frac{i}{N}\right)
$$

for some positive real number $M_{N}$ and continuously differentiable functions $\sigma_{\tau}$ and $\tilde{\sigma}_{\tau}$, (H1) and (H2) are also fulfilled provided $M_{N}-\alpha N$ converges towards a constant $c(\alpha)$ and

$$
\sup _{\tau \in \Omega}\left\|\sigma_{\tau}^{\prime}\right\|_{u}<\infty, \quad \text { and } \quad \sup _{\tau \in \Omega}\left\|\tilde{\sigma}_{\tau}^{\prime}\right\|_{u}<\infty .
$$

(H1) is satisfied with $c(\Delta(\phi, \tilde{\phi}))=2^{-1}(\phi(1)+\tilde{\phi}(\alpha)-\phi(0)-\tilde{\phi}(0))+c(\alpha) \tilde{\phi}(\alpha)$. Remark that if $M_{N}$ is an integer number, the first assumption should only be valid along subsequences in general. We can choose $\mathcal{D}$ to be

$$
\mathcal{D}_{d}^{\prime}=\left\{\Delta: \Delta=\Delta(\phi, \tilde{\phi}) \text { for some } \phi, \tilde{\phi} \in \mathcal{C}_{b}^{1}([0,1], \mathbb{R})\right\} .
$$

To state our large deviation upper bound result, we have to be more precise about the involved topologies and space of measures.

$\mathcal{M}$ is furnished with the operator norm; if $\langle,\rangle_{N}$ denotes the Euclidean scalar product in $\mathbb{C}^{N},\langle u, v\rangle_{N}=\sum_{i=1}^{N} \bar{u}_{i} v_{i}$, and \|\|$_{N}$ its associated norm, we define the operator norm | $\left.\right|_{\infty}$ by setting, for any $A \in \mathcal{M}_{N}, N \in \mathbb{N}$,

$$
|A|_{\infty}=\sup _{\|u\|_{N}=1}\langle u,|A| u\rangle_{N}=\sup _{\|u\|_{N}=1}\left\langle u, A A^{*} u\right\rangle_{N}^{\frac{1}{2}} .
$$

Recall that ||$_{\infty}$ is a norm which satisfies the product property

$$
|A B|_{\infty} \leqslant|A|_{\infty}|B|_{\infty} .
$$

$\mathcal{M}$ is furnished with the involution *, extension of the usual involution on each $\mathcal{M}_{N}$, $N \in \mathbb{N}$. Also, there is a partial order on $\mathcal{H}$ so that $A \leqslant B$ for $A, B \in \mathcal{H}_{N}, N \in \mathbb{N}$, iff $\langle u, A u\rangle_{N} \leqslant\langle u, B u\rangle_{N}$ for all $u \in \mathbb{C}^{N}$. 
We can endow $\mathcal{E}(\mathbb{C})$ with the topology inherited from the norm given for any $F \in \mathcal{E}(\mathbb{C})$, by

$$
\|F\|_{\infty}=\sup _{N \geqslant 1} \sup \left\{|F(A)|_{\infty}: A \in \mathcal{H}_{N}\right\} .
$$

It is not hard to check (see [10], Lemma 4.26) that, with (2.2),

Lemma (2.11). - Any $F \in F_{\mathbb{C}}(X, \mathcal{D})$ has finite \|\|$_{\infty}$ norm.

We let $\mathcal{F}_{\mathbb{C}}(X, \mathcal{D})\left(\right.$ resp. $\left.\mathcal{F}_{\mathbb{R}}(X, \mathcal{D})\right)$ be the completion of $F_{\mathbb{C}}(X, \mathcal{D})\left(\right.$ resp. $\left.F_{\mathbb{R}}(X, \mathcal{D})\right)$ by the \|\|$_{\infty}$ norm. $\mathcal{F}_{\mathbb{C}}(X, \mathcal{D})\left(\right.$ resp. $\mathcal{F}_{\mathbb{R}}(X, \mathcal{D})$ ) is a complex (resp. real) Banach space. Further, they are separable. In fact, since $\mathcal{D}$ was assumed separable (remark that the norm defined on $\mathbb{D}$ agrees with \|\|$\left._{\infty}\right), \mathcal{F}_{\mathbb{C}}(X, \mathcal{D})$ is separable for \|\|$_{\infty}$ with a basis given, for instance, by the set of functions of the form

$$
F(X)=\prod_{1 \leqslant j \leqslant n}\left(i+\alpha_{j}-\alpha_{j}^{\prime} X\right)^{-1} \Delta_{j}(X), \quad X \in \mathcal{H}, \Delta_{j} \in \mathcal{B}_{\mathcal{D}}, \alpha_{j}, \alpha_{j}^{\prime} \in \mathbb{Q}, n \in \mathbb{N},
$$

if $\mathcal{B}_{\mathcal{D}}$ is a basis of $\mathcal{D}$.

We can now define the set of non-commutative probability measures; let $\mathcal{F}_{\mathbb{C}}(X, \mathcal{D})^{\prime}$ be the algebraic dual of $\mathcal{F}_{\mathbb{C}}(X, \mathcal{D})$, that is the space of linear complex-valued forms on $\mathcal{F}_{\mathbb{C}}(X, \mathcal{D})$. Let $\mathcal{M}$ be the subset of $\mathcal{F}_{\mathbb{C}}(X, \mathcal{D})^{\prime}$ with real valued restriction to $\mathcal{F}_{\mathbb{R}}(X, \mathcal{D})$. $\mathcal{M}$ is isomorphic to $\mathcal{F}_{\mathbb{R}}(X, \mathcal{D})^{\prime}$ since for any $\mu \in \mathcal{M}$, we can write, with * the natural involution defined by

$$
\begin{gathered}
F^{*}(X)=(F(X))^{*} \quad \forall X \in \mathcal{H}, \\
\mu(F)=\mu\left(\frac{F+F^{*}}{2}\right)+i \mu\left(\frac{F-F^{*}}{2 i}\right),
\end{gathered}
$$

where $\left(F+F^{*}\right)$ and $\left(F-F^{*}\right) / i \in \mathcal{F}_{\mathbb{R}}(X, \mathcal{D})$. We furnish $\mathcal{M}$ with the weak topology induced by $\mathcal{F}_{\mathbb{R}}(X, \mathcal{D})$, denoted by $\mathcal{F}_{\mathbb{R}}(X, \mathcal{D})$-topology.

We shall now introduce the analogue of the set of probability measures (that is the notions of boundedness, positivity and mass 1 ).

For any positive real number $a$, we denote by $\mathcal{M}_{a}$ the subset of $\mathcal{M}$ of linear forms $\mu$ such that

$$
\forall F \in \mathcal{F}_{\mathbb{C}}(X, \mathcal{D}), \quad|\mu(F)| \leqslant a\|F\|_{\infty} .
$$

Further, let us consider the following partial order on $\mathcal{E}(\mathbb{R})$; If $(F, G) \in \mathcal{E}(\mathbb{R}), F \leqslant G$ iff

$$
\forall X \in \mathcal{H}, \quad G(X) \geqslant F(X) .
$$

We shall say that a linear form $\mu \in \mathcal{M}$ is positive iff

$$
\forall F \in \mathcal{F}_{\mathbb{R}}(X, \mathcal{D}) \quad F \geqslant 0 \Longrightarrow \mu(F) \geqslant 0 .
$$

$\mu$ will be said to be tracial if

$$
\forall F, G \in \mathcal{F}_{\mathbb{C}}(X, \mathcal{D}) \quad \mu(G F)=\mu(F G) .
$$


Let $\mathcal{M}_{a}^{+}$be the subset of $\mathcal{M}_{a}$ of positive tracial linear forms. We can define the notion of total mass for any linear form $\mu$ of $\mathcal{M}_{a}^{+}$by

$$
m_{\mu}=\sup \left\{\mu(F), F \in \mathcal{F}_{\mathbb{R}}(X, \mathcal{D}),\|F\|_{\infty} \leqslant 1\right\}=\mu(1)
$$

The analogue of the commutative set of probability measures will be the subset $\mathcal{M}_{1}^{=}$of $\mathcal{M}_{1}^{+}$of linear form with total mass $m_{\mu}$ exactly equal to one.

By a standard diagonalization procedure, it is not hard to check as in the commutative setting that $\mathcal{M}_{1}^{=}$is compact for the $\mathcal{F}_{\mathbb{R}}(X, \mathcal{D})$-topology since $\mathcal{F}_{\mathbb{R}}(X, \mathcal{D})$ is separable. The $\mathcal{F}_{\mathbb{R}}(X, \mathcal{D})$-topology is compatible on $\mathcal{M}_{1}^{=}$with the distance

$$
\bar{d}(\mu, \nu)=\||| \mu-v||\left|\equiv \sum_{p \in \mathbb{N}} \frac{1}{2^{p}}\right| \mu\left(F_{p}\right)-v\left(F_{p}\right) \mid,
$$

where $\left(F_{p}\right)_{p \in \mathbb{N}}$ is a basis of uniformly bounded functions of $F_{\mathbb{R}}(X, \mathcal{D})$ as described in (2.12). Hence, $\mathcal{M}_{1}^{=}$is a compact metric space, thus Polish.

Let $\hat{\mu}_{t}^{(N)}$ be given by

$$
\hat{\mu}_{t}^{(N)}(F)=\operatorname{tr}_{N}\left(F\left(X_{N}(t)\right)\right) \quad \forall F \in \mathcal{F}_{\mathbb{R}}(X, \mathcal{D}), \forall t \in[0,1] .
$$

Then, considering $\left(\hat{\mu}_{t}^{(N)}, 0 \leqslant t \leqslant 1\right)$ as a continuous $\mathcal{M}_{1}^{=}$-valued process and endowing the set $\mathcal{C}\left([0,1], \mathcal{M}_{1}^{=}\right)$of such processes with the uniform topology on the time variable and the $\mathcal{F}_{\mathbb{R}}(X, \mathcal{D})$-topology on $\mathcal{M}_{1}^{=}$, we shall prove that

THEOREM (2.14). - Under (H0), the law of $\left(\hat{\mu}_{t}^{(N)}, 0 \leqslant t \leqslant 1\right)$ satisfies a large deviation upper bound in the scale $N^{2}$ with good rate function $S$ described in Theorem (4.1).

We discuss in Section 4 after Theorem (4.1) the large deviation upper bound obtained by contraction from Theorem (2.14) for the law of $\hat{\mu}_{1}^{(N)}$ and its relation with the noncommutative entropy introduced by D. Shlyakhtenko.

Let us make a few remarks about the corollaries of Theorem (2.14) in terms of standard large deviation principle. Since we discussed this point in details in [10], we shall here be rather sketchy. To this end, we recall the links of $\mathcal{M}_{1}^{=}$with standard spaces of probability measures. It is based on the following remark of [10] (see Property 4.32 and Lemma 4.26) that

PROPERTY (2.15). - Let $F \in \mathcal{F}_{\mathbb{R}}(X, \mathcal{D})$.

(1) For any $f \in \mathcal{C}_{b}(\mathbb{R}), f \circ F$ belongs to $\mathcal{F}_{\mathbb{R}}(X, \mathcal{D})$.

(2) The linear functional $\mu_{F}$ on $\mathcal{C}_{b}(\mathbb{R})$ given by

$$
\mu_{F}(f)=\mu(f \circ F)
$$

is a compactly supported probability measure on $\mathbb{R}$ for any $\mu \in \mathcal{M}_{1}^{=}$. Further, the map $\mu \rightarrow \mu_{F}$ from $\mathcal{M}_{1}^{=}$, furnished with the $\mathcal{F}_{\mathbb{R}}(X, \mathcal{D})$-topology, into $\mathcal{P}(\mathbb{R})$, furnished with the weak topology, is continuous.

As a consequence, the contraction principle and Theorem (2.14) imply 
COROLlaRY (2.16). - Let $F \in \mathcal{F}_{\mathbb{R}}(X, \mathcal{D})$. Then, the spectral measure process of $\left(F\left(X_{N}(t)\right), t \in[0,1]\right)$ satisfies a large deviation upper bound for the weak topology in the scale $N^{2}$ with good rate function $S_{F}$ given for any $v \in \mathcal{C}([0,1], \mathcal{P}(\mathbb{R}))$ by

$$
S_{F}(v)=\inf \left\{S(\mu) ;\left(\mu_{F}\right)_{t}=v_{t} \forall t \in[0,1]\right\} .
$$

Note that at this point, we do not obtain a large deviation upper bound for the spectral process of $X_{N}$ itself since $F(X)=X$ does not belong to $\mathcal{F}_{\mathbb{R}}(X, \mathcal{D})$. To get such a result, we shall prove in addition a tightness criterium which requires the next observations. As in [10], we can define a probability measure $\mu_{X}$ on $\mathbb{R}$ so that for any $f \in \mathcal{C}_{b}(\mathbb{R}), \mu_{X}(f)=\mu(f(X))$. In particular, $\mu_{X}$ is countably additive and the monotone convergence theorem holds $[21,1.26]$. Hence, we can set $\mu\left(X^{2}\right)=\mu_{X}\left(x^{2}\right)$. Let, for $A \in \mathbb{R}^{+}, \mathcal{K}_{1}^{=}(A)$ be the closed subset of $\mathcal{M}_{1}^{=}$

$$
\mathcal{K}_{1}^{=}(A) \equiv\left\{\mu \in \mathcal{M}_{1}^{=}, \mu_{X}\left(x^{2}\right) \leqslant A\right\}
$$

and

$$
\mathcal{K}_{1}^{=}(\infty) \equiv \bigcup_{A \in \mathbb{N}} \mathcal{K}_{1}^{=}(A)=\left\{\mu \in \mathcal{M}_{1}^{=}, \mu_{X}\left(x^{2}\right)<\infty\right\} .
$$

In Theorem (4.1), $\hat{\mu}^{(N)}$ is considered as an element of $\mathcal{C}\left([0,1], \mathcal{M}_{1}^{=}\right)$but we see that all the $\hat{\mu}_{t}^{(N)}$ belong to $\mathcal{K}_{1}^{=}(A)$ with probability as large as we wish on the exponential scale provided $A$ is large enough (but finite). Also, the processes with entropy $S$ smaller than some $M$ are shown to have covariance uniformly bounded by some constant depending on $M$. This is enough to see that the $\mathcal{F}_{\mathbb{R}}(X, \mathcal{D})$-topology will be equivalent in our setting with the topology obtained by duality of the set

$$
\overline{\mathcal{F}}_{\mathbb{R}}(X, \mathcal{D}) \equiv\left\{F \in \mathcal{E}(\mathbb{R}) ; \exists\left(F_{n}\right)_{n \in \mathbb{N}} \in \mathcal{F}_{\mathbb{R}}(X, \mathcal{D})^{\mathbb{N}},\left|F-F_{n}\right|(X) \leqslant \frac{1}{n}\left(X^{2}+1\right)\right\},
$$

where $|F(X)|=\sqrt{F(X)^{2}} \cdot \overline{\mathcal{F}}_{\mathbb{R}}(X, \mathcal{D})$ contains the canonical process $X$ (approximate $X$ by $\left.X\left(1+n^{-2} X^{2}\right)^{-1} \in \mathcal{F}_{\mathbb{R}}(X, \mathcal{D})\right)$. More precisely, we have the following extension of Property (2.15):

Lemma (2.17). - Let $F \in \overline{\mathcal{F}}_{\mathbb{R}}(X, \mathcal{D})$ and $\mu \in \mathcal{K}_{1}^{=}(A)$ for some $A \in \mathbb{R}^{+}$. Then, we can define

$$
\mu_{F}(f)=\lim _{n \rightarrow \infty} \mu_{F_{n}}(f), \quad f \in \mathcal{C}_{b}(\mathbb{R}) .
$$

$\mu_{F}$ is a probability measure on $\mathbb{R}$. Moreover, the map $\mu \rightarrow \mu_{F}$ is continuous from $\mathcal{K}_{1}^{=}(A)$ into $\mathcal{P}(\mathbb{R})$ for any $A \in \mathbb{R}^{+}$.

The proof is the same as that of Property 4.33 in [10].

As a consequence, using Theorem (4.1) and standard exponential approximations described in [12], Section 4.2.2 (see the proof of Corollary 4.4 of [10] for details) we obtain

COROLlaRY (2.19). - The conclusions of Corollary (2.16) are valid for any $F \in$ $\overline{\mathcal{F}}_{\mathbb{R}}(X, \mathcal{D})$. 
To complete this introduction, we wish to summarize two applications. First, let us consider the band matrix given by the model studied in this paper with

$$
\psi_{N}(i, j)=\psi\left(\frac{i}{N}, \frac{j}{N}\right)=\int \sigma_{\tau}\left(\frac{i}{N}\right) \sigma_{\tau}\left(\frac{j}{N}\right) \mathrm{d} p(\tau)
$$

for bounded continuous functions $\sigma_{\tau}$. As quoted in Examples (2.4), we can choose $\mathcal{D}=\mathcal{D}_{c}$. With such a choice, the law of large number statements (2.5), (2.6) as well as the large deviation upper bounds results (2.14), (2.16) and (2.19) apply. For the central limit theorem, under the hypothesis of Examples (2.9), we can take $\mathcal{D}=\mathcal{D}_{c}^{\prime}$ and conclude.

We can also apply our results to the generalized Gaussian Wishart's matrices given by

$$
W^{N}=Y^{N} T^{N}\left(Y^{N}\right)^{*}
$$

with $Y^{N}$ a $N \times M_{N}$ complex Gaussian matrix with independent entries of covariance $\frac{1}{N}$ and $T^{N}$ a $M_{N} \times M_{N}$ diagonal matrix with non-negative eigenvalues. As in [14], we observe that $W^{N}$ is related to band matrices as follows. If $X_{N}$ is given by

$$
X_{N}=\left(\begin{array}{cc}
0 & Y^{N}\left(T^{N}\right)^{\frac{1}{2}} \\
\left(T^{N}\right)^{\frac{1}{2}}\left(Y^{N}\right)^{*} & 0
\end{array}\right),
$$

the spectrum of $\left(X_{N}\right)^{2}$ is given by the spectrum of $W^{N}$ with multiplicity two up to some null eigenvalues since

$$
\left(X_{N}\right)^{2}=\left(\begin{array}{cc}
Y^{N} T^{N}\left(Y^{N}\right)^{*} & 0 \\
0 & \left(T^{N}\right)^{\frac{1}{2}}\left(Y^{N}\right)^{*} Y^{N}\left(T^{N}\right)^{\frac{1}{2}}
\end{array}\right) .
$$

Further, $X_{N}$ has the law of

$$
\left(\psi_{N}(i, j)^{\frac{1}{2}} H^{N+M_{N}}(1)_{i j}\right)_{1 \leqslant i, j \leqslant N+M_{N}}
$$

with, if $t_{1}, \ldots, t_{M_{N}}$ denote the eigenvalues of $T^{N}$,

$$
\psi_{N}(i, j) \equiv 1_{N+1 \leqslant i \leqslant N+M_{N}} 1_{1 \leqslant j \leqslant N} t_{i}+1_{N+1 \leqslant j \leqslant N+M_{N}} 1_{1 \leqslant i \leqslant N} t_{j} .
$$

We assume for simplification that $t_{i}=t\left(\frac{i}{N+M_{N}}\right)$ for some bounded continuous non negative function $t$. Notice that $\psi_{N}$ can be written

$$
\psi_{N}(i, j)=\int \sigma_{\tau}^{\alpha_{N}}\left(\frac{i}{N+M_{N}}\right) \sigma_{\tau}^{\alpha_{N}}\left(\frac{j}{N+M_{N}}\right) \mathrm{d} p(\tau)
$$

with $p(\tau)=\delta_{\tau=1}-\delta_{\tau=2}-\delta_{\tau=3}, \alpha_{N}=(N+1)\left(N+M_{N}\right)^{-1}$ and, for $\alpha \in \mathbb{R}^{+}, x \in[0,1]$,

$$
\begin{aligned}
& \sigma_{1}^{\alpha}(x)=1_{x \geqslant \alpha}(t(x)-1)+1, \\
& \sigma_{2}^{\alpha}(x)=1_{x \geqslant \alpha} t(x), \\
& \sigma_{3}^{\alpha}(x)=1-1_{x \geqslant \alpha} .
\end{aligned}
$$


Hence, following example (b) given in (2.4), if $\frac{N+1}{N+M_{N}}$ converges as $N$ goes to infinity towards a constant $\alpha$ and if we choose $\mathcal{D}$ to be $\mathcal{D}_{d}$ described in Examples (2.4) (b), the results (2.5), (2.6), (2.14), (2.16) and (2.19) apply to $X_{N}$. We denote by $S_{T}$ the rate function governing the large deviation of the spectral measure process $\hat{\mu}^{(N)}$ in the scale $\left(N+M_{N}\right)^{2}$ coming from Theorem (2.14) with the above specific choice of measure $p$ and functions $\left(\sigma_{i}^{\alpha}\right)_{1 \leqslant i \leqslant 3}$. To deduce the same results for $W^{N}$, observe that, if $\Delta_{3}^{N}=\Delta\left(\sigma_{3}^{\alpha_{N}}\right)$,

$$
\Delta_{3}^{N}\left(X_{N}\right)^{2} \Delta_{3}^{N}=\left(\begin{array}{cc}
W^{N} & 0 \\
0 & 0
\end{array}\right)
$$

It is not hard to see that for any $F \in \mathcal{F}_{\mathbb{C}}(X, \mathcal{D})$,

$$
G(X)=F\left(\Delta_{3} X^{2} \Delta_{3}\right) \in \mathcal{F}_{\mathbb{C}}(X, \mathcal{D}),
$$

since for any $z \in \mathbb{C} \backslash \mathbb{R}$ we can write

$$
\left(z-\Delta_{3} X^{2} \Delta_{3}\right)^{-1}=\Delta_{3}\left(z^{\frac{1}{2}}+X\right)^{-1}\left(z^{\frac{1}{2}}-X\right)^{-1} \Delta_{3}+\left(I-\Delta_{3}\right) z^{-1}
$$

with any choice of the square root $z^{\frac{1}{2}}$ of $z$. Hence, if $\mu_{T}$ is defined by

$$
\mu_{T}(F)=\mu\left(F\left(\Delta_{3} X^{2} \Delta_{3}\right)\right), \quad F \in \mathcal{F}_{\mathbb{C}}(X, \mathcal{D}),
$$

the map $\mu \rightarrow \mu_{T}$ in $\mathcal{M}_{1}^{=}$, furnished with the $\mathcal{F}_{\mathbb{R}}(X, \mathcal{D})$-topology, is continuous. We can hence deduce from the contraction principle and Theorem (2.14) the following result. Set

$$
\hat{\mu}_{T, t}^{(N)}(F)=\operatorname{tr}_{N}\left(F\left(W_{t}^{N}\right)\right), \quad F \in \mathcal{F}_{\mathbb{C}}(X, \mathcal{D}),
$$

with $W_{t}^{N}$ constructed as $W^{N}$ but with Brownian motion entries.

COROLLARY (2.23). - Assume that $(N+1) /\left(N+M_{N}\right)$ converges towards a positive constant $\alpha$. Then, the law of $\hat{\mu}_{T, .}^{(N)}$ satisfies a large deviation upper bound in the scale $\left(N+M_{N}\right)^{2}$ for the $\mathcal{F}_{\mathbb{R}}(X, \mathcal{D})$-topology. The good rate function governing this large deviations upper bound is given by

$$
\begin{gathered}
I_{T}(\mu)=\inf \left\{S_{T}(v) ;\right. \\
v_{1}\left(F\left(\Delta_{3} X^{2} \Delta_{3}\right)\right)=\mu(F)+v_{0}\left(\left(I-\Delta_{3}\right) F\left(I-\Delta_{3}\right)\right) \\
\left.\forall F \in \mathcal{F}_{\mathbb{R}}(X, \mathcal{D})\right\} .
\end{gathered}
$$

A large deviation upper bound in the weak topology for the law of the spectral measure of $W_{N}$ can of course be deduced from Corollary (2.23) by the contraction principle. We then refer to [15] for a full large deviation principle when the eigenvalues of $T^{N}$ are uniformly bounded below by a positive constant. The central limit theorem for polynomial functions of $X^{N}$ and $W^{N}$ can also be deduced from Theorem (2.8) under the hypothesis that $N+1-\alpha\left(N+M_{N}\right)$ converges as underlined in Examples (2.9). This hypothesis is needed to insure the convergence of the expectation of $N\left(\operatorname{tr}_{N}-\mu_{1}^{*}\right) P$ but would not be required if we would consider the fluctuations of the spectral measure around its mean.

The central ingredient to prove the previous theorems is an Itô's formula for $\left(\hat{\mu}_{t}^{(N)}, t \in\right.$ $[0,1])$. We shall prove it in the next section. 


\section{Itô's calculus}

To present the stochastic differential calculus for the process $X_{N}$, we need first to define a few differential operators. Most of them can be already encountered in [10] where the reader can find a more detailed introduction.

\subsection{Differential operators}

Let us first recall the definition of the non-commutative derivation. It is the linear map $D_{X}$ from $F_{\mathbb{C}}(X, \mathcal{D})$ into $\mathcal{E}(\mathbb{C}) \otimes \mathcal{E}(\mathbb{C})$ so that for any $F, G \in F_{\mathbb{C}}(X, \mathcal{D})$,

$$
\lim _{\varepsilon \rightarrow 0} \varepsilon^{-1}(F(X+\varepsilon G(X))-F(X))=D_{X} F \sharp G(X)
$$

with the notation $(A \otimes B) \sharp C=A C B$ and where $\otimes$ denotes the standard tensor product. $D_{X}$ can be equivalently described by the the non-commutative Leibnitz rule and its action on basic functionals. The non-commutative Leibnitz rule says that for every $F, G \in F_{\mathbb{C}}(X, \mathcal{D})$, any $A \in \mathcal{H}$,

$$
D_{X}(F G)(A)=D_{X}(F)(A) \times 1 \otimes G(A)+F(A) \otimes 1 \times D_{X}(G)(A) .
$$

Here $\times$ denotes the multiplication in the tensor product space so that for any $N \in \mathbb{N}$, any $A, B, C, D \in \mathcal{M}_{N}, A \otimes B \times C \otimes D=A C \otimes B D$. Then, $D_{X}$ is uniquely defined if we set for any $A \in \mathcal{H}$, any $z \in \mathbb{C} \backslash \mathbb{R}$, any $\alpha \in \mathbb{R}$,

$$
D_{X}\left(\frac{1}{z-\alpha X}\right)(A)=\alpha \frac{1}{z-\alpha A} \otimes \frac{1}{z-\alpha A}
$$

and for any $\Delta \in \mathcal{D}$,

$$
D_{X}(\Delta)(A)=0 .
$$

Notice that

$$
D_{X}\left(F_{\mathbb{C}}(X, \mathcal{D})\right) \subset F_{\mathbb{C}}(X, \mathcal{D}) \otimes F_{\mathbb{C}}(X, \mathcal{D}) .
$$

We can thus define a second order operator $D_{X}^{2}$ from $F_{\mathbb{C}}(X, \mathcal{D}) \otimes F_{\mathbb{C}}(X, \mathcal{D})$ into $F_{\mathbb{C}}(X, \mathcal{D}) \otimes F_{\mathbb{C}}(X, \mathcal{D}) \otimes F_{\mathbb{C}}(X, \mathcal{D})$ by

$$
D_{X}^{2} \equiv \frac{1}{2}\left(D_{X} \otimes 1+1 \otimes D_{X}\right) \circ D_{X} .
$$

Let, for $\tau \in \Omega, M_{\tau}$ be the map from $\mathcal{M}_{N} \otimes \mathcal{M}_{N} \otimes \mathcal{M}_{N}$ into $\mathcal{M}_{N} \otimes \mathcal{M}_{N}$ for any $N \in \mathbb{N}$ so that for any $A, B, C \in \mathcal{M}_{N}$,

$$
M_{\tau}(A \otimes B \otimes C) \equiv \Delta_{\tau}^{N} B \otimes A \Delta_{\tau}^{N} C
$$

for any $N \in \mathbb{N}$. We set for $X \in \mathcal{H}$,

$$
\left(\mathbb{L}_{\tau} F\right)(X) \equiv M_{\tau}\left(D_{X}^{2} F(X)\right)
$$


and

$$
(\mathbb{L} F)(X)=\int\left(\mathbb{L}_{\tau} F\right)(X) \mathrm{d} p(\tau) .
$$

It is also natural to define the derivation $\mathcal{D}_{X}$ from $F_{\mathbb{C}}(X, \mathcal{D})$ into $\mathcal{E}(\mathbb{C})$ so that for any trace $\mu \in \mathcal{M}_{1}^{=}$, any $F, G \in F_{\mathbb{C}}(X, \mathcal{D})$,

$$
\lim _{\varepsilon \rightarrow 0} \varepsilon^{-1} \mu(F(X+\varepsilon G(X))-F(X))=\mu\left(\mathcal{D}_{X} F \times G(X)\right) .
$$

$\mathcal{D}_{X}$ is often called the cyclic derivative. It was already noticed in [10] that if $m$ is the map from $\mathcal{M}_{N} \otimes \mathcal{M}_{N}$ into $\mathcal{M}_{N}$ for all $N \in \mathbb{N}$, so that $m(A \otimes B)=B A$,

$$
\mathcal{D}_{X}=m \circ D_{X} .
$$

Also, in view of (3.1) and since $F_{\mathbb{C}}(X, \mathcal{D})$ is an algebra,

$$
\mathcal{D}_{X}\left(F_{\mathbb{C}}(X, \mathcal{D})\right) \subset F_{\mathbb{C}}(X, \mathcal{D}) .
$$

We also set $\mathcal{D}^{*}$ to be the linear operator on $F_{\mathbb{C}}(X, \mathcal{D})$ so that

$$
\mathcal{D}^{*}(F)(X)=(\mathcal{D} F(X))^{*} \quad \forall X \in \mathcal{H} .
$$

Finally, if we let $m_{\tau}: \mathcal{M}_{N} \rightarrow \mathcal{M}_{N}$ for all $N \in \mathbb{N}$ be the left-hand side multiplication by $\Delta_{\tau}$, that is for any $A \in \mathcal{H}_{N}, N \in \mathbb{N}$,

$$
m_{\tau}(A) \equiv \Delta_{\tau}^{N} A,
$$

we set

$$
\mathcal{L}_{\tau} \equiv \frac{1}{2} m_{\tau} \otimes m_{\tau} \circ D_{X} \circ \mathcal{D}_{X} .
$$

Then, we define the operator from $F_{\mathbb{C}}(X, \mathcal{D})$ into $F_{\mathbb{C}}(X, \mathcal{D}) \otimes F_{\mathbb{C}}(X, \mathcal{D})$

$$
\mathcal{L}=\int \mathcal{L}_{\tau} \mathrm{d} p(\tau)
$$

that is that for every test function $F \in F_{\mathbb{C}}(X, \mathcal{D})$, any $A \in \mathcal{H}$,

$$
\mathcal{L} F(A)=\int\left(\mathcal{L}_{\tau} F\right)(A) \mathrm{d} p(\tau)
$$

\subsection{Itô's formula}

Let $\mathcal{C}^{1}\left([0,1], F_{\mathbb{R}}(X, \mathcal{D})\right)$ be the set of time-continuously differentiable functions with values in $F_{\mathbb{R}}(X, \mathcal{D})$ and time derivative in $F_{\mathbb{R}}(X, \mathcal{D})$. We next show the

LEMMA (3.5). -

(1) Itô's formula for the matrix-valued process $X_{N}$ : for every $F \in \mathcal{C}^{1}([0,1]$, $\left.F_{\mathbb{C}}(X, \mathcal{D})\right)$, any $t \in[0,1]$, 


$$
\begin{aligned}
F_{t}\left(X_{N}(t)\right)= & F_{0}\left(X_{N}(0)\right)+\int_{0}^{t} \operatorname{tr}_{N} \otimes \operatorname{Id}\left(\mathbb{L}\left(F_{s}\right)\left(X_{N}(s)\right)\right) \mathrm{d} s \\
& +\int_{0}^{t} \partial_{s} F_{s}\left(X_{N}(s)\right) \mathrm{d} s+\int_{0}^{t} D_{X} F\left(X_{N}(s)\right) \sharp \mathrm{d} X_{N}(s) .
\end{aligned}
$$

(2) Itô's formula for the measure-valued process: for every $F \in \mathcal{C}^{1}\left([0,1], F_{\mathbb{R}}(X, \mathcal{D})\right)$, any $t \in[0,1]$,

$$
\begin{aligned}
Q_{F}^{(N)}(t)= & \operatorname{tr}_{N} F_{t}\left(X_{N}(t)\right)-\operatorname{tr}_{N} F_{0}\left(X_{N}(0)\right)-\int_{0}^{t} \operatorname{tr}_{N}\left[\partial_{s} F_{s}\left(X_{N}(s)\right)\right] \mathrm{d} s \\
& -\int_{0}^{t}\left(\operatorname{tr}_{N} \otimes \operatorname{tr}_{N}\right)\left[\mathcal{L} F_{s}\left(X_{N}(s)\right)\right] \mathrm{d} s
\end{aligned}
$$

is a real-valued martingale with bracket

$$
\left\langle Q_{F}^{(N)}\right\rangle_{t}=\frac{1}{N^{2}} \int_{0}^{t} \int \operatorname{tr}_{N}\left[m_{\tau}\left(\mathcal{D}_{X} F_{S}\left(X_{N}(s)\right)\right) m_{\tau}\left(\mathcal{D}_{X}^{*} F_{S}\left(X_{N}(s)\right)\right)\right] \mathrm{d} p(\tau) \mathrm{d} s .
$$

Proof. - The proof follows multi-dimensional Itô's formula. Indeed, considering $F_{t}\left(X_{N}\right)$ as a function of the entries of $H_{N}$, remark that for any $i, j \in\{1, \ldots, N\}$, and with $\left(X_{N}\right)_{i j}=h_{i j} \psi_{N}(i, j)^{\frac{1}{2}}$,

$$
\partial_{h_{i j}} F_{t}\left(X_{N}\right)=D_{X} F_{t}\left(X_{N}\right) \sharp\left(\partial_{h_{i j}} X_{N}\right),
$$

and for any $k, l \in\{1, \ldots, N\}$,

$$
\begin{aligned}
\partial_{h_{k l}} \partial_{h_{i j}} F_{t}\left(X_{N}\right)= & D_{X} \otimes 1 \circ D_{X} F_{t}\left(X_{N}\right) \sharp\left(\partial_{h_{k l}} X_{N}, \partial_{h_{i j}} X_{N}\right) \\
& +1 \otimes D_{X} \circ D_{X} F_{t}\left(X_{N}\right) \sharp\left(\partial_{h_{i j}} X_{N}, \partial_{h_{k l}} X_{N}\right),
\end{aligned}
$$

where we have denoted by $A \otimes B \otimes C \sharp(D, E)=A D B E C$. Also, remark that

$$
\left(\partial_{h_{i j}} X_{N}\right)_{k l}=\delta_{i j=k l} \psi_{N}(i, j)^{\frac{1}{2}}
$$

Now, recall that multi-dimensional Itô's calculus yields, since $\left\langle\left(H_{N}\right)_{k l},\left(H_{N}\right)_{i j}\right\rangle_{t}=$ $N^{-1} \delta_{k l=j i} t$,

$$
\begin{aligned}
\mathrm{d} F_{t}\left(X_{N}(t)\right)= & \partial_{t} F_{t}\left(X_{N}(t)\right) \mathrm{d} t+\sum_{i, j=1}^{N} \partial_{h_{i j}} F_{t}\left(X_{N}(t)\right)\left(\mathrm{d} X_{N}(t)\right)_{i j} \\
& +\frac{1}{2 N} \sum_{i, j=1}^{N} \psi_{N}(i, j) \partial_{h_{j i}} \partial_{h_{i j}} F_{t}\left(X_{N}(t)\right) \mathrm{d} t
\end{aligned}
$$

But, according to (3.7), for any $k, l, m, n, o, p \in\{1, \ldots, N\}$, if we denote by $(A \otimes B \otimes$ $C)_{k l m n o p}=A_{k l} B_{m n} C_{o p}$, 


$$
\begin{aligned}
& \left(\frac{1}{2 N} \sum_{i, j=1}^{N} \psi_{N}(i, j) \partial_{h_{j i}} \partial_{h_{i j}} F_{t}(X)\right)_{k l} \\
& \quad=\frac{1}{N} \sum_{i, j=1}^{N} \psi_{N}(i, j)\left(D_{X}^{2} F\right)_{k i j j i l} \\
& \quad=\int\left(\frac{1}{N} \sum_{i, j=1}^{N} \sigma_{\tau}^{N}(i) \sigma_{\tau}^{N}(j)\left(D_{X}^{2} F\right)_{k i j j i l}\right) \mathrm{d} p(\tau) \\
& \quad=\int\left(\operatorname{tr}_{N} \otimes \operatorname{Id}\left(\mathbb{L}_{\tau} F(X)\right)\right)_{k l} \mathrm{~d} p(\tau)
\end{aligned}
$$

giving the first part of the lemma.

For the second part, we need only to take the trace on both sides of (3.6) to obtain

$$
\begin{aligned}
\mathrm{d} \operatorname{tr}_{N} F_{t}\left(X_{N}(t)\right)= & \operatorname{tr}_{N}\left(\partial_{t} F_{t}\left(X_{N}(t)\right)\right) \mathrm{d} t+\operatorname{tr}_{N}\left(D_{X} F\left(X_{N}(t)\right) \sharp \mathrm{d} X_{N}(t)\right) \\
& +\int \operatorname{tr}_{N} \otimes \operatorname{tr}_{N}\left(\mathbb{L}_{\tau} F\left(X_{N}(t)\right)\right) \mathrm{d} p(\tau) \mathrm{d} t .
\end{aligned}
$$

The first term in (3.8) gives the martingale term

$$
\operatorname{tr}_{N}\left(D_{X} F_{t}\left(X_{N}(t)\right) \sharp \mathrm{d} X_{N}(t)\right)=\operatorname{tr}_{N}\left(\mathcal{D}_{X} F_{t}\left(X_{N}(t)\right) \mathrm{d} X_{N}(t)\right) .
$$

For the second term, observe that

$$
\operatorname{tr}_{N} \otimes \operatorname{tr}_{N}\left(\mathbb{L}_{\tau} F(X)\right)=\operatorname{tr}_{N} \otimes \operatorname{tr}_{N}\left(\mathcal{L}_{\tau} F(X)\right) .
$$

Indeed, denoting $\left(F_{i}^{1}, F_{i}^{2}\right)$ a family of functions in $F_{\mathbb{C}}(X, \mathcal{D})$ so that $D_{X} F=\sum_{i} F_{i}^{1} \otimes$ $F_{i}^{2}$, we find that

$$
D_{X}^{2} F=\frac{1}{2} \sum_{i} \sum_{j}\left(\left(F_{i}^{1}\right)_{j}^{1} \otimes\left(F_{i}^{1}\right)_{j}^{2} \otimes F_{i}^{2}+F_{i}^{1} \otimes\left(F_{i}^{2}\right)_{j}^{1} \otimes\left(F_{i}^{2}\right)_{j}^{2}\right)
$$

so that

$$
M_{\tau}\left(D_{X}^{2} F\right)=\frac{1}{2} \sum_{i} \sum_{j}\left(\Delta_{\tau}\left(F_{i}^{1}\right)_{j}^{2} \otimes\left(F_{i}^{1}\right)_{j}^{1} \Delta_{\tau} F_{i}^{2}+\Delta_{\tau}\left(F_{i}^{2}\right)_{j}^{1} \otimes F_{i}^{1} \Delta_{\tau}\left(F_{i}^{2}\right)_{j}^{2}\right) .
$$

On the other hand,

$$
\begin{aligned}
\mathcal{L}_{\tau} F & =\frac{1}{2} m_{\tau} \otimes m_{\tau} \circ D_{X}\left(\sum_{i} F_{i}^{2} F_{i}^{1}\right) \\
& =\frac{1}{2} \sum_{i} \sum_{j}\left(\Delta_{\tau}\left(F_{i}^{1}\right)_{j}^{2} \otimes \Delta_{\tau} F_{i}^{2}\left(F_{i}^{1}\right)_{j}^{1}+\Delta_{\tau}\left(F_{i}^{2}\right)_{j}^{1} \otimes \Delta_{\tau}\left(F_{i}^{2}\right)_{j}^{2} F_{i}^{1}\right)
\end{aligned}
$$

so that taking the trace satisfying

$$
\operatorname{tr}_{N}\left(\Delta_{\tau}\left(F_{i}^{2}\right)_{j}^{2} F_{i}^{1}\right)=\operatorname{tr}_{N}\left(F_{i}^{1} \Delta_{\tau}\left(F_{i}^{2}\right)_{j}^{2}\right)
$$


gives (3.11). Hence,

$$
Q_{F}^{(N)}(t)=\operatorname{tr}_{N}\left(\mathcal{D}_{X} F_{t}\left(X_{N}(t)\right) \mathrm{d} X_{N}(t)\right)
$$

is a martingale. Its bracket is easily computed by

$$
\left\langle\left(\mathrm{d} X_{N}\right)_{i j},\left(\mathrm{~d} X_{N}\right)_{k l}\right\rangle_{t}=\delta_{i j=l k} N^{-1} \psi_{N}(i, j) \mathrm{d} t .
$$

The fact that the martingale is real valued is clear since, as $F_{t} \in F_{\mathbb{R}}(X, \mathcal{D})$,

$$
\operatorname{tr}_{N}\left(F_{t}\right)=\operatorname{tr}_{N}\left(F_{t}^{*}\right)=\overline{\operatorname{tr}_{N}\left(F_{t}\right)}
$$

since $\operatorname{tr}_{N}$ is invariant by transposition.

\section{Large deviation upper bound}

We shall prove a large deviation upper bound for non-commutative functionals of the process of $\left(X_{N}(t)\right)_{t \in[0,1]}$ in this section. The rate function for these deviations is defined as follows. First, we define the empty state $\delta_{0}$ to be the element of $\mathcal{K}_{1}^{=}(\infty)$ so that for any $F \in F_{\mathbb{R}}(X, \mathcal{D}), F(X)=\prod_{1 \leqslant i \leqslant n}\left(z_{i}-\alpha_{i} X\right)^{-1} \Delta_{i}$,

$$
\delta_{0}(F)=m(F(0)),
$$

where $m(F(0))$ is defined by (2.3) since $F(0)=\prod_{1 \leqslant i \leqslant n} z_{i}^{-1} \Delta_{i} \in \mathcal{D}$ by construction. We let $\mathcal{C}_{s}\left([0,1], \mathcal{M}_{1}^{=}\right)$be the subset of $\mathcal{C}\left([0,1], \mathcal{M}_{1}^{=}\right)$of continuous $\mathcal{M}_{1}^{=}$-valued processes $\mu$ so that $\mu_{0}=\delta_{0}$ and for any $\Delta \in \mathcal{D}$, any $t \in[0,1]$,

$$
\mu_{t}(\Delta)=\delta_{0}(\Delta) \text {. }
$$

Then, $S$ is defined by

$$
S(\mu)= \begin{cases}+\infty & \text { if } \mu \notin \mathcal{C}_{s}\left([0,1], \mathcal{M}_{1}^{=}\right), \\ \sup _{0 \leqslant s \leqslant t \leqslant 1} S^{s, t}(\mu) & \text { otherwise, }\end{cases}
$$

with, if for $F, G \in \mathcal{C}^{1}\left([0,1], \mathcal{F}_{\mathbb{R}}(X, \mathcal{D})\right)$, we define for any times $0 \leqslant s \leqslant t \leqslant 1$, any $\mu \in \mathcal{C}\left([0,1], \mathcal{M}_{1}^{=}\right)$,

$$
\begin{gathered}
S^{s, t}(F, \mu)=\mu_{t}\left(F_{t}\right)-\mu_{s}\left(F_{s}\right)-\int_{s}^{t} \mu_{u}\left(\partial_{u} F_{u}\right) \mathrm{d} u-\int_{s}^{t} \mu_{u}\left(\mathcal{L} F_{u}\right) \mathrm{d} u, \\
\langle\langle F, G\rangle\rangle_{\mu}^{s, t}=\int_{s}^{t} \int \mu_{u}\left(m_{\tau}\left(\mathcal{D}_{X} F_{u}\right) m_{\tau}\left(\mathcal{D}_{X}^{*} G_{u}\right)\right) \mathrm{d} p(\tau) \mathrm{d} u, \\
S^{s, t}(\mu)=\sup _{F \in \mathcal{C}^{1}\left([0,1], F_{\mathbb{R}}(X, \mathcal{D})\right)}\left(S^{s, t}(F, \mu)-\frac{1}{2}\langle\langle F, F\rangle\rangle_{\mu}^{s, t}\right) .
\end{gathered}
$$


Let us denote by $\hat{\mu}_{t}^{(N)}$ the linear map on $\mathcal{F}_{\mathbb{C}}(X, \mathcal{D})$ so that for any $F \in \mathcal{F}_{\mathbb{C}}(X, \mathcal{D})$, any $t \in[0,1]$,

$$
\hat{\mu}_{t}^{(N)}(F)=\operatorname{tr}_{N}\left(F\left(X_{N}(t)\right)\right) .
$$

We infer that $\hat{\mu}^{(N)}$ belongs to $\mathcal{C}\left([0,1], \mathcal{M}_{=}^{1}\right)$. We shall prove in this section that

THEOREM (4.1). - The law of $\hat{\mu}^{(N)} \in \mathcal{C}\left([0,1], \mathcal{M}_{=}^{1}\right)$ satisfies a large deviation upper bound in the scale $N^{2}$ with good rate function $S$, that is

(1) $S$ is a non-negative function with compact level sets for the $\mathcal{F}_{\mathbb{R}}(X, \mathcal{D})$-topology.

Further, for any $M>0$, there exists a $A>0$ so that

$$
E_{M}=\{S \leqslant M\} \subset \mathcal{C}\left([0,1], \mathcal{K}_{1}^{=}(A)\right) .
$$

(2)

$$
\limsup _{A \rightarrow \infty} \limsup _{N \rightarrow \infty} \frac{1}{N^{2}} \log \mathbb{P}\left(\hat{\mu}^{(N)} \in \mathcal{C}\left([0,1], \mathcal{K}_{1}^{=}(A)\right)^{c}\right)=-\infty .
$$

(3) For any closed subset $F$ of $\mathcal{C}\left([0,1], \overline{\mathcal{M}}_{=}^{1}\right)$,

$$
\limsup _{N \rightarrow \infty} \frac{1}{N^{2}} \log \mathbb{P}\left(\hat{\mu}^{(N)} \in F\right) \leqslant-\inf _{F} S .
$$

In particular, since the application $\mu \in \mathcal{C}\left([0,1], \mathcal{M}_{=}^{1}\right) \rightarrow \mu_{1} \in \mathcal{M}_{=}^{1}$ is continuous, we deduce from the contraction principle that

COROLlaRY (4.2). $-\hat{\mu}_{1}^{(N)}$ satisfies a large deviation upper bound in the scale $N^{2}$ with good rate function given for $\mu \in \mathcal{M}_{=}^{1}$ by

$$
S_{1}(\mu)=\inf \left\{S(v) ; v \in \mathcal{C}\left([0,1], \mathcal{M}_{=}^{1}\right): v_{1}=\mu\right\} .
$$

It is natural that the above infimum should be achieved at the limit process $\mu^{b}$ obtained by conditioning the entries at time 1 . It satisfies the differential equation

$$
\partial_{t} \mu_{t}^{b}(F)=-\mu_{t}^{b} \otimes \mu_{t}^{b}(\mathcal{L} F)+\mu_{t}^{b}\left(\frac{X}{t} \mathcal{D}_{X} F\right) .
$$

$\mu_{t}^{b}$ can also be constructed as the law of $t A+X_{t(1-t)}$ where $\left(X_{s}, s \in[0,1]\right)$ is the limit of $\left(X_{N}(s), s \in[0,1]\right)$ and $A$ has law $\mu_{1}$ and is free from $\left(X_{N}(s), s \in[0,1]\right)$. We then deduce an upper bound for $S_{1}$ given by

$$
S_{1}(\mu) \leqslant S\left(\mu^{b}\right) \leqslant \int_{0}^{1} u^{-1} J\left(\tilde{\mu}_{u}^{b}\right) \mathrm{d} u
$$

with $J$ the Fisher's information given, if $\|F\|_{\mu}^{2}:=\int \mu\left(m_{\tau}(F) m_{\tau}\left(F^{*}\right)\right) \mathrm{d} p(\tau)$, by

$$
J(\mu)=\sup _{F \in F_{\mathbb{R}}(X, \mathcal{D})}\left\{2 \mu \otimes \mu(\mathcal{L} F)-\mu\left(X \mathcal{D}_{X} F\right)-\frac{1}{2}\left\|\mathcal{D}_{X} F\right\|_{\mu}^{2}\right\}
$$


and $\tilde{\mu}_{u}^{b}$ the image of $\mu_{u}^{b}$ by the homothety of ratio $u^{-\frac{1}{2}}$. By a translation on the function $F$, we find

$J(\mu)=\sup _{F \in F_{\mathbb{R}}(X, \mathcal{D})}\left\{\mu \otimes \mu\left(\int \mathrm{d} p(\tau) m_{\tau} \otimes m_{\tau} D_{X} \circ \mathcal{D}_{X} F\right)-\frac{1}{2}\left\|\mathcal{D}_{X} F\right\|_{\mu}^{2}\right\}+\frac{1}{2} \mu\left(X^{2}\right)-1$.

Thus, $J(\mu)$ is finite iff $\mu\left(X^{2}\right)<\infty$ and, by Riesz's theorem, if there exists $H \in$

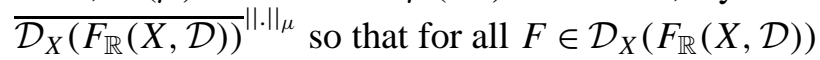

$$
\mu \otimes \mu\left(\int \mathrm{d} p(\tau) \Delta_{\tau} \otimes \Delta_{\tau} \times D_{X} F\right)=\int \mu\left(m_{\tau}(F) m_{\tau}\left(H^{*}\right)\right) \mathrm{d} p(\tau),
$$

and then

$$
J(\mu)=\frac{1}{2}\|H\|_{\mu}^{2}+\frac{1}{2} \mu\left(X^{2}\right)-1 .
$$

Thus, the natural Fisher entropy is here given in terms of the image by the adjoint of $D_{X}$ of $\int \mathrm{d} p(\tau) \Delta_{\tau} \otimes \Delta_{\tau}$ (compare with Wigner's matrices where one takes the image of $1 \otimes 1$ by the same adjoint (see [25])). This Fisher's entropy is related to that defined by D. Shlyakhtenko [24].

The proof of this theorem follows the usual scheme; we first study the rate function $S$ and prove that it is a good rate function. We then show that $\hat{\mu}^{(N)}$ is exponentially tight and provide then a weak large deviation upper bound.

\subsection{Study of the rate function}

LEMMA (4.3). $-S$ is a non-negative function which has compact level sets for the $\mathcal{F}_{\mathbb{R}}(X, \mathcal{D})$-topology. Further, for any $M>0$, there exists a $A>0$ so that

$$
E_{M}=\{S \leqslant M\} \subset \mathcal{C}\left([0,1], \mathcal{K}_{1}^{=}(A)\right) .
$$

Proof. - First notice that $S$ is non-negative since, for $\mu$ with $S(\mu)<\infty$, we have

$$
\begin{aligned}
S(\mu) & =\sup _{0 \leqslant s \leqslant t \leqslant 1} \sup _{F \in \mathcal{C}^{1}\left([0,1], F_{\mathbb{R}}(X, \mathcal{D})\right)}\left(S^{s, t}(F, \mu)-\frac{1}{2}\langle\langle F, F\rangle\rangle_{\mu}^{s, t}\right) \\
& =\sup _{0 \leqslant s \leqslant t \leqslant 1} \sup _{F \in \mathcal{C}^{1}\left([0,1], F_{\mathbb{R}}(X, \mathcal{D})\right)} \sup _{\lambda \in \mathbb{R}}\left(\lambda S^{s, t}(F, \mu)-\frac{\lambda^{2}}{2}\langle\langle F, F\rangle\rangle_{\mu}^{s, t}\right) \\
& =\frac{1}{2} \sup _{0 \leqslant s \leqslant t \leqslant 1} \sup _{F \in \mathcal{C}^{1}\left([0,1], F_{\mathbb{R}}(X, \mathcal{D})\right)} \frac{\left(S^{s, t}(F, \mu)\right)^{2}}{\langle\langle F, F\rangle\rangle_{\mu}^{s, t}}
\end{aligned}
$$

is nonnegative as $S^{s, t}(F, \mu),\langle\langle F, F\rangle\rangle_{\mu}^{s, t} \in \mathbb{R} \times \mathbb{R}^{+}$. Further, for any $F \in \mathcal{C}^{1}([0,1]$, $\left.F_{\mathbb{R}}(X, \mathcal{D})\right), \mu \rightarrow S^{s, t}(F, \mu)$ is continuous by the stability properties of (3.1)-(3.4). For the same reason, $\mu \rightarrow\langle\langle F, F\rangle\rangle_{\mu}^{s, t}$ is continuous and hence $S^{0,1}$, as a supremum of continuous functions, is lower semi-continuous that is has closed level sets. Since $\mathcal{M}_{1}^{=}$is compact, the precompact subsets of $\mathcal{C}^{1}\left([0,1], \mathcal{M}_{=}^{1}\right)$ can be included in compact sets of the form $\mathcal{K}=\bigcap_{n \in \mathbb{N}} \mathcal{K}_{n}^{\prime}$ with $\mathcal{K}_{n}^{\prime}=\left\{v \in \mathcal{C}\left([0,1], \mathcal{M}_{1}^{=}\right) \mid\right.$the function $(u \rightarrow$ 
$\left.v_{u}\left(F_{n}\right)\right)$ belongs to $\left.K_{n}^{\prime}\right\}$ if $\left(K_{n}^{\prime}\right)_{n \in \mathbb{N}}$ a sequence of compact subsets of $\mathcal{C}_{b}([0,1], \mathbb{R})$ and $\left(F_{n}\right)_{n \in \mathbb{N}}$ a basis of $F_{\mathbb{R}}(X, \mathcal{D})$. In view of Arzéla-Ascoli theorem, the compact subsets $K^{\prime}$ of $\mathcal{C}([0,1], \mathbb{R})$ are such that there exists a finite constant $C>0$, a family $\varepsilon_{n}$ of positive real numbers $\varepsilon_{n}, \varepsilon_{n} \rightarrow 0$ as $n$ goes to infinity, a family of positive real numbers $\delta_{n}$ such that

$$
K^{\prime}=\left\{f \in \mathcal{C}_{b}([0,1], \mathbb{R}),\|f\|_{u} \leqslant C, \sup _{|t-s| \leqslant \delta_{n}}|f(t)-f(s)| \leqslant \varepsilon_{n}, \forall n \in \mathbb{N}\right\} .
$$

Hence, to prove that the level sets $E_{M}$ can be included into some $\mathcal{K}$, we need to show that for every $F \in F_{\mathbb{R}}(X, \mathcal{D})$, and every $m>0$, there exists $\delta_{m}^{M}(F)$ so that

$$
\forall v \in E_{M}, \sup _{|t-s| \leqslant \delta_{m}^{M}(F)}\left|v_{t}(F)-v_{s}(F)\right| \leqslant \frac{1}{m} .
$$

Since by definition we have for all $F \in F_{\mathbb{R}}(X, \mathcal{D})$, for any $v \in E_{M}$,

$$
S^{s, t}(\nu, F)^{2} \leqslant 2 S^{0,1}(v)\langle\langle F, F\rangle\rangle_{v}^{s, t} \leqslant 2 M\langle\langle F, F\rangle\rangle_{v}^{s, t}
$$

we deduce

$$
\left|v_{t}(F)-v_{s}(F)\right| \leqslant\left|\int_{s}^{t} v_{u} \otimes v_{u}(\mathcal{L} F) \mathrm{d} u\right|+\sqrt{2 M\langle\langle F, F\rangle\rangle_{v}^{s, t}} .
$$

By definition of $F_{\mathbb{R}}(X, \mathcal{D}),(3.1)-(3.4)$ and Lemma (2.11), all the functions appearing in the above right-hand side are uniformly bounded for \|\|$_{\infty}$ so that we conclude that there exists a finite constant $C^{M}(F)$ such that

$$
\left|v_{t}(F)-v_{s}(F)\right| \leqslant C^{M}(F)(\sqrt{|t-s|}+|t-s|) .
$$

Finally, to prove (4.4), we take

$$
F(X)=\frac{X^{2}}{1+\varepsilon X^{2}}=\frac{X}{i+\sqrt{\varepsilon} X} \frac{X}{-i+\sqrt{\varepsilon} X} \in F_{\mathbb{R}}(X, \mathcal{D})
$$

and compute $\mathcal{D}_{X} F(X)=2 X\left(1+\varepsilon X^{2}\right)^{-2}$, resulting with

$$
\begin{aligned}
\mathcal{L} F(X, X)= & \int \Delta_{\tau} \otimes\left(\Delta_{\tau}\left(1+\varepsilon X^{2}\right)^{-2}\right) \mathrm{d} p(\tau) \\
& +\int \Delta_{\tau} X \otimes \Delta_{\tau} \times D_{X}\left(1+\varepsilon X^{2}\right)^{-2} \mathrm{~d} p(\tau) .
\end{aligned}
$$

Further, $X \otimes 1 D_{X}\left(1+\varepsilon X^{2}\right)^{-2}$ is given by

$$
\varepsilon \sum_{n=0,1 ; p=1,2}\left(\left(1+\varepsilon X^{2}\right)^{-p} X^{n}\right) \otimes\left(\left(1+\varepsilon X^{2}\right)^{-3+p} X^{1-n}\right),
$$


and therefore is easily checked to be the sum of tensor product of bounded operators with norm bounded above independently of $\varepsilon$. As a consequence, $\mathcal{L} F$ can be uniformly bounded in the tensor product space, independently of $\varepsilon$.

Hence, there exists a finite constant $C$ so that if $\mu \in E_{M}$, for all $t \in[0,1]$,

$$
\mu_{t}(F) \leqslant M+C+\int_{0}^{t} \mu_{s}\left(\left(\Delta_{\tau} \frac{2 X}{\left(1+\varepsilon X^{2}\right)^{2}}\right)^{2}\right) \mathrm{d} s .
$$

It is not hard to verify that by the trace and positivity properties of $\mu_{s}$, CauchySchwartz's inequality type statements are valid and that $\forall F, G \in \mathcal{F}_{\mathbb{R}}(X, \mathcal{D}), F \geqslant 0$,

$$
\mu_{s}(G F) \leqslant\|G\|_{\infty} \mu_{s}(F) .
$$

Hence, we compute

$$
\mu_{s}\left(\left(\Delta_{\tau} \frac{2 X}{\left(1+\varepsilon X^{2}\right)^{2}}\right)^{2}\right) \leqslant 4\left\|\Delta_{\tau}\right\|_{\infty}^{2} \mu_{s}\left(\left(\frac{X}{\left(1+\varepsilon X^{2}\right)^{2}}\right)^{2}\right) \leqslant 4\left\|\Delta_{\tau}\right\|_{\infty}^{2} \mu_{s}(F)
$$

so that we conclude, since the operator norm of $\Delta_{\tau}$ is uniformly bounded by $T$ by assumption (H0), that

$$
\mu_{t}(F) \leqslant(C+M)+4 T^{2} \int_{0}^{t} \mu_{s}(F) \mathrm{d} s
$$

and hence by Gronwall's lemma

$$
\sup _{t \in[0,1]} \mu_{t}(F) \leqslant(C+M) \mathrm{e}^{4 T^{2}} .
$$

We can now let $\varepsilon \downarrow 0$ and conclude that $\sup _{t \in[0,1]} \mu\left(X_{t}^{2}\right) \leqslant\left(T^{2}+M\right) \mathrm{e}^{4 T^{2}}$ which proves the second point of the lemma.

\subsection{Exponential tightness}

Lemma (4.7). - There exists compact subsets $\mathcal{K}_{L}, L \in \mathbb{N}$, of $\mathcal{C}\left([0,1], \mathcal{M}_{1}^{=}\right)$so that

$$
\limsup _{N \rightarrow \infty} \frac{1}{N^{2}} \log \mathbb{P}\left(\hat{\mu}^{(N)} \in \mathcal{K}_{L}^{c}\right) \leqslant-L .
$$

The proof follows the description of the precompact sets $\mathcal{C}\left([0,1], \mathcal{M}_{1}^{=}\right)$given in the last part and is given in details in [10] in a slightly different context. We shall not detail it here. Further,

LEMMA (4.8). -

$$
\underset{A \rightarrow \infty}{\limsup } \limsup _{N \rightarrow \infty} \frac{1}{N^{2}} \log \mathbb{P}\left(\hat{\mu}^{(N)} \in \mathcal{C}\left([0,1], \mathcal{K}_{A}\right)^{c}\right)=-\infty .
$$


Proof. - This amounts to prove that

$$
\limsup _{A \rightarrow \infty} \limsup _{N \rightarrow \infty} \frac{1}{N^{2}} \log \mathbb{P}\left(\sup _{t \in[0,1]} \hat{\mu}_{t}^{(N)}\left(X^{2}\right) \geqslant A\right)=-\infty .
$$

But

$$
\begin{aligned}
\sup _{t \in[0,1]} \hat{\mu}_{t}^{(N)}\left(X^{2}\right) & =\sup _{t \in[0,1]} \frac{1}{N} \sum_{i, j=1}^{N} \psi_{N}(i, j)\left|H_{N}(t)_{i, j}\right|^{2} \\
& \leqslant \frac{1}{N^{2}} \sum_{i, j=1}^{N} \psi_{N}(i, j) \sup _{t \in[0,1]}\left(\left(\beta_{t}^{i, j}\right)^{2}+\left(\tilde{\beta}_{t}^{i, j}\right)^{2}\right) .
\end{aligned}
$$

Since $\psi_{N}$ is uniformly bounded and Désiré-André reflection principle ensures that $\sup _{t \in[0,1]}\left(\beta_{t}^{i, j}\right)^{2}$ has some finite exponential moments, we find an $\alpha>0$ and a finite constant $C_{\alpha}$ so that

$$
\mathbb{P}\left[\mathrm{e}^{\alpha N^{2} \sup _{t \in[0,1]} \hat{\mu}_{t}^{(N)}\left(X^{2}\right)}\right] \leqslant C_{\alpha}^{N^{2}}
$$

which, thanks to Chebyshev's inequality, allows us to conclude.

\subsection{Weak large deviation upper bound}

In view of Lemma (4.7), we can get a large deviation upper bound by means of a weak large deviation upper bound which is an easy consequence of

LEMMA (4.9). -

$$
\underset{\delta \downarrow 0}{\limsup } \limsup _{N \rightarrow \infty} \frac{1}{N^{2}} \log \mathbb{P}\left(\overline{\mathcal{D}}\left(v, \hat{\mu}^{(N)}\right)<\delta\right) \leqslant-S(v)
$$

for any $v \in \mathcal{C}\left([0,1], \mathcal{M}_{1}^{=}\right)$.

Proof. - Note that, at time 0 ,

$$
\hat{\mu}_{0}^{(N)}(F)=\operatorname{tr}_{N}(F(0))
$$

converges, as $F(0) \in \mathcal{D}$, towards $m(F(0))$ by (H0). Thus, for any $\eta>0$, for $N$ large enough $d\left(\hat{\mu}_{0}^{(N)}, \delta_{0}\right) \leqslant \delta$. Hence, with $\hat{\mu}_{t}^{(N)}(F)=\hat{\mu}_{0}^{(N)}(F)$ for any $F \in \mathcal{D}$, we deduce that

$$
\limsup _{\delta \downarrow 0} \limsup _{N \rightarrow \infty} \frac{1}{N^{2}} \ln \mathbb{P}\left(\hat{\mu}^{(N)} \in B_{\delta}(v)\right)=-\infty
$$

if $v \notin \mathcal{C}_{s}\left([0,1], \mathcal{M}_{1}^{=}\right)$. Therefore, we shall assume hereafter that $v \in \mathcal{C}_{s}\left([0,1], \mathcal{M}_{1}^{=}\right)$. We shall follow the ideas developed in [19]. To this end, we define a family of positives super-martingales $\left\{\zeta_{F}^{(N)}, F \in \mathcal{C}^{1}\left([0,1], F_{\mathbb{R}}(X, \mathcal{D})\right)\right\}$, equal to 1 at $t=0$, thanks to Lemma (3.5):

$$
\zeta_{F}^{(N)}(t)=\exp \left(N^{2} Q_{F}^{(N)}(t)-\frac{N^{4}}{2}\left\langle Q_{F}^{(N)}\right\rangle_{t}\right)
$$




$$
=\exp \left(N^{2}\left(S^{0, t}\left(\hat{\mu}^{(N)}, F\right)-\frac{1}{2}\langle\langle F, F\rangle\rangle_{\hat{\mu}^{(N)}}^{0, t}\right)\right) .
$$

Let $v \in \mathcal{C}\left([0,1], \mathcal{M}_{1}^{=}\right)$and $F \in \mathcal{C}\left([0,1], F_{\mathbb{R}}(X, \mathcal{D})\right)$; then for any $0 \leqslant s \leqslant t \leqslant 1$, if $\zeta_{F}^{(N)}(t, s)=\zeta_{F}^{(N)}(t) \zeta_{F}^{(N)}(s)^{-1}$,

$$
\begin{aligned}
\mathbb{P}\left(\hat{\mu}^{(N)} \in B(v, \delta)\right) & =\mathbb{E}\left[1_{\hat{\mu}^{(N)} \in B(v, \delta)} \frac{\zeta_{F}^{(N)}(t, s)}{\zeta_{F}^{(N)}(t, s)}\right] \\
& \leqslant \sup _{v^{\prime} \in B(v, \delta)} \exp \left(-N^{2}\left(S^{s, t}\left(v^{\prime}, F\right)-\frac{1}{2}\langle\langle F, F\rangle\rangle_{v^{\prime}}^{s, t}\right)\right) \\
& =\exp \left(-N^{2} \inf _{v^{\prime} \in B(v, \delta)}\left(S^{s, t}\left(v^{\prime}, F\right)-\frac{1}{2}\langle\langle F, F\rangle\rangle_{v^{\prime}}^{s, t}\right)\right),
\end{aligned}
$$

where we have used $\mathbb{E}\left[\zeta_{F}^{(N)}(t, s)\right] \equiv 1$. Notice that if $F$ belongs to $\mathcal{C}^{1}\left([0,1], F_{\mathbb{R}}(X, \mathcal{D})\right)$, the function $v^{\prime} \rightarrow S^{0,1}\left(v^{\prime}, F\right)-\frac{1}{2}\langle\langle F, F\rangle\rangle_{v^{\prime}}^{0,1}$ is continuous. Thus, for any function $F \in \mathcal{C}^{1}\left([0,1], F_{\mathbb{R}}(X, \mathcal{D})\right)$

$$
\limsup _{\delta \downarrow 0} \limsup _{N \rightarrow \infty} \frac{1}{N^{2}} \ln \mathbb{P}\left(\hat{\mu}^{(N)} \in B(\nu, \delta)\right) \leqslant-\left(S^{0,1}(\nu, F)-\frac{1}{2}\langle\langle F, F\rangle\rangle_{\nu}^{0,1}\right) .
$$

We conclude by taking the supremum over $F$ that

$$
\limsup _{\delta \downarrow 0} \limsup _{N \rightarrow \infty} \frac{1}{N^{2}} \ln \mathbb{P}\left(\hat{\mu}^{(N)} \in B(v, \delta)\right) \leqslant-S(v) .
$$

\section{Law of large numbers}

According to the large deviation upper bound of the previous section, we know that $\hat{\mu}^{(N)}$, as an element of $\mathcal{C}\left([0,1], \mathcal{M}_{1}^{=}\right)$, concentrates almost surely towards the minimizers of $S$. In this section, we prove that $S$ admits a unique minimizer and study it. We then deduce a law of large numbers theorem for bounded test functions which we strengthen in a second time to include polynomial functions.

\subsection{Study of the minimizers of $S$}

Since $S$ is a good rate function, it achieves its minimum value, which is zero. Its minimizers are hence characterized as the $\mu \in \mathcal{C}_{s}\left([0,1], \mathcal{M}_{1}^{=}\right)$satisfying

$$
S^{0,1}(\mu, F)=0
$$

for all test functions $F$. We shall prove that

LEMMA (5.2). - (5.1) admits a unique solution $\mu^{*} \in \mathcal{C}_{s}\left([0,1], \mathcal{M}_{=}^{1}\right)$.

To prove Lemma (5.2), we first show that the minimizers have finite moments and provide bounds for them; 
Lemma (5.3). - There exists a finite constant $C$ so that if $\mu$ minimizes $S$,

$$
\sup _{t \in[0,1]} \mu_{t}\left(X^{2 n}\right) \leqslant n ! C^{n}, \quad \forall n \in \mathbb{N} .
$$

In particular, as a standard probability measure, $\mu_{t}$ is defined by its moments.

Proof. - Set, for $\varepsilon>0, F(X)=\frac{X^{2}}{1+\varepsilon X^{2}}$. Following (4.5), we have

$$
\mathcal{D}_{X} F^{n}(X)=2 n F^{n-1}(X) \frac{X}{\left(1+\varepsilon X^{2}\right)^{2}}
$$

and

$$
\begin{aligned}
D_{X} \circ \mathcal{D}_{X} F^{n}(X)= & 4 n \sum_{\xi=0,1} \sum_{k=0}^{n-2} F^{k}(X) \frac{X^{\xi}}{\left(1+\varepsilon X^{2}\right)} \otimes \frac{X^{1-\xi}}{\left(1+\varepsilon X^{2}\right)} F^{n-2-k}(X) \frac{X}{\left(1+\varepsilon X^{2}\right)^{2}} \\
& +2 n F^{n-1}(X) \otimes 1 \times D_{X} \circ \mathcal{D}_{X} F(X) .
\end{aligned}
$$

Noticing that $\left\|\Delta_{\tau}\right\|_{\infty} \leqslant T$ and for any $k \in \mathbb{N}$,

$$
\mu\left(F^{k} \frac{X}{\left(1+\varepsilon X^{2}\right)}\right) \leqslant \mu\left(F^{k} \frac{X^{2}+1}{2\left(1+\varepsilon X^{2}\right)}\right) \leqslant \frac{1}{2}\left(\mu\left(F^{k}\right)+\mu\left(F^{k+1}\right)\right),
$$

and recalling from (4.6) that $D_{X} \circ \mathcal{D}_{X} F$ is uniformly bounded in the tensor product space, we find a finite constant $C$ so that for any $\mu \in \mathcal{M}_{1}^{=}$,

$$
\mu \otimes \mu\left(\mathcal{L} F^{n}\right) \leqslant C n \sum_{k=0}^{n-2} \mu\left(F^{k+1}+F^{k}\right) \mu\left(F^{n-k-1}+F^{n-k-2}\right) .
$$

Hence, if $\mu$ satisfies (5.1), and $m_{n}(t) \equiv \sup _{\varepsilon \in[0,1]} \sup _{0 \leqslant k \leqslant n} \mu_{t}\left(F^{k}\right)$, we have

$$
m_{n}(t) \leqslant 1+\left(4 n^{2} C\right) \int_{0}^{t} m_{n-1}(s)^{2} \mathrm{~d} s,
$$

so that with $m_{n}=\sup _{t \in[0,1]} m_{n}(t) \geqslant 1$,

$$
m_{n} \leqslant\left(4 n^{2} C+1\right) m_{n-1}^{2} \leqslant \prod_{p=1}^{n}\left(4 p^{2} C+1\right)^{2^{n-p}} .
$$

Hence,

$$
\mu\left(X^{2 n}\right)=\lim _{\varepsilon \downarrow 0} \mu\left[\left(\frac{X^{2}}{1+\varepsilon X^{2}}\right)^{n}\right]
$$

exists and is finite for all $n$. We can therefore extend (5.1) by talking $F$ to be polynomial. We then get the easier formula 


$$
\begin{aligned}
& \partial_{t} \mu_{t}\left(\prod_{1 \leqslant i \leqslant n}^{\rightarrow} \Delta_{i} X\right)=\sum_{k=1}^{n} \sum_{l=0}^{n-k-1} \int \mathrm{d} p(\tau) \mu_{t}\left(\Delta_{\tau} \prod_{k+1 \leqslant i \leqslant k+l} \Delta_{i} X \Delta_{k+l+1}\right) \\
& \times \mu_{t}\left(\Delta_{\tau} \prod_{k+l+2 \leqslant i \leqslant n} \Delta_{i} X \prod_{1 \leqslant i \leqslant k-1}^{\rightarrow} \Delta_{i} X \Delta_{k}\right) \\
& +\sum_{k=1}^{n} \sum_{l=1}^{k-1} \int \mathrm{d} p(\tau) \mu_{t}\left(\Delta_{\tau} \prod_{k+1 \leqslant i \leqslant n}^{\rightarrow} \Delta_{i} X \prod_{1 \leqslant i \leqslant l}^{\rightarrow} \Delta_{i} X \Delta_{l}\right) \\
& \times \mu_{t}\left(\Delta_{\tau} \prod_{l+1 \leqslant i \leqslant k-1} \Delta_{i} X \Delta_{k}\right)
\end{aligned}
$$

for any $\left(\Delta_{1}, \ldots, \Delta_{n}\right) \in \mathcal{D}$. By induction over $n$, we deduce that $\mu_{t}\left(\prod_{1 \leqslant i \leqslant n} \Delta_{i} X\right)=0$ if $n$ is odd for every $\Delta_{i} \in \mathcal{D}$. Taking $\Delta_{i}=1$, we get if $n$ is even,

$$
\partial_{t} \mu_{t}\left(X^{n}\right) \leqslant T^{2} n \sum_{k=0}^{n-2 / 2} \mu_{t}\left(X^{2 k}\right) \mu_{t}\left(X^{n-2 k-2}\right)
$$

Let

$$
u_{n}(t)=\frac{1}{n !} \mu_{t}\left(X^{2 n}\right)
$$

Then, (5.6) implies

$$
\partial_{t} u_{n}(t) \leqslant T^{2} \sum_{k=0}^{n-1}\left(C_{n-1}^{k}\right)^{-1} u_{k}(t) u_{n-1-k}(t)
$$

with $C_{n}^{k}=(n ! / k !(n-k) !)$. Observe that $c=\sup _{n \in \mathbb{N}} \sum_{k=0}^{n-1}\left(C_{n-1}^{k}\right)^{-1}<\infty$ so that by induction we see that there exists $C<\infty\left(C \leqslant c T^{2}\right)$ such that

$$
\sup _{t \in[0,1]} u_{n}(t) \leqslant C^{n}
$$

which finishes the proof of the lemma.

We are now in position to prove Lemma (5.2):

Proof of Lemma (5.2). - Finally, the moments of $\mu_{t}$ are uniquely determined since, if $\mu, v$ are two solutions,

$$
\Delta_{k}(t)=\sup _{n \leqslant k} \sup _{\Delta \in \mathcal{D},\|\Delta\|_{\infty} \leqslant 1}\left|v_{t}\left(\prod_{1 \leqslant i \leqslant n}^{\rightarrow} \Delta_{i} X\right)-\mu_{t}\left(\prod_{1 \leqslant i \leqslant n}^{\rightarrow} \Delta_{i} X\right)\right|
$$

we have by the above equation

$$
\Delta_{k}(t) \leqslant 2 k^{2} \sqrt{k ! C^{k}} T^{2} \int_{0}^{t} \Delta_{k-1}(s) \mathrm{d} s \leqslant 2 k^{2} \sqrt{k ! C^{k}} T^{2} \int_{0}^{t} \Delta_{k}(s) \mathrm{d} s
$$


which, by Gronwall's lemma implies $\Delta_{k}(t)=0$. In view of Lemma (5.3), this is enough to guarantee directly that for any $\xi_{1}, \ldots, \xi_{n} \in \mathbb{C}, z_{1}, \ldots, z_{i} \in \mathbb{C}$

$$
\mu_{t}\left(\prod_{1 \leqslant i \leqslant n}^{\rightarrow} \Delta_{i} \mathrm{e}^{\xi_{i}\left(X-z_{i}\right)}\right)
$$

is uniquely defined (check that the expansion indeed converges) and then by integration over the $\xi_{k}$ 's when $\Im\left(z_{k}\right) \neq 0$ (with $\operatorname{sgn}\left(\Im\left(z_{k}\right)\right) \xi_{k} \in(-\infty, 0]$ ), that $\mu_{t}(F)$ are uniquely determined for $F \in F_{\mathbb{C}}(X, \mathcal{D})$, insuring the uniqueness of $\mu_{t}$ as an element of $\mathcal{M}_{1}^{=}$for any $t \in[0,1]$.

Let us notice that $\left(\mu_{t}^{*}, t \in[0,1]\right)$ satisfies a scaling property

Lemma (5.7). - For any $t \in[0,1]$, iffor $F \in F_{\mathbb{C}}(X, \mathcal{D}), F^{t}(X)=F(\sqrt{t} X)$,

$$
\mu_{t}^{*}(F)=\mu_{1}^{*}\left(F^{t}\right) .
$$

Proof. - Indeed, $\mathcal{L}$, as a second order differential operator on $X$, satisfies for any $F \in F_{\mathbb{C}}(X, \mathcal{D})$,

$$
\mathcal{L} F^{t}(X)=t(\mathcal{L} F)(\sqrt{t} X) .
$$

Hence, since $\mu_{t}^{*}$ is uniquely characterized by (5.1), we have for any $t \in[0,1], \lambda \in$ $\left(0, t^{-1}\right]$, for any function $F \in F_{\mathbb{C}}(X, \mathcal{D})$

$$
\begin{aligned}
\mu_{\lambda t}^{*}(F) & =\delta_{0}(F)+\int_{0}^{\lambda t} \mu_{s}^{*} \otimes \mu_{s}^{*}(\mathcal{L} F) \mathrm{d} s \\
& =\delta_{0}(F)+\int_{0}^{t} \mu_{\lambda s}^{*} \otimes \mu_{\lambda s}^{*}(\lambda \mathcal{L} F) \mathrm{d} s \\
& =\delta_{0}(F)+\int_{0}^{t} \mu_{\lambda s}^{*} \otimes \mu_{\lambda s}^{*}\left(\left(\mathcal{L} F^{\lambda}\right)\left(\sqrt{\lambda}^{-1} X\right)\right) \mathrm{d} s
\end{aligned}
$$

Thus, $\left(\mu_{t}^{\lambda}, t \in[0,1]\right)$ given by

$$
\mu_{t}^{\lambda}(F)=\mu_{\lambda t}^{*}\left(F\left(\sqrt{\lambda}^{-1} X\right)\right), \quad F \in F_{\mathbb{C}}(X, \mathcal{D})
$$

satisfies

$$
\mu_{t}^{\lambda}(F)=\delta_{0}(F)+\int_{0}^{t} \mu_{s}^{\lambda} \otimes \mu_{s}^{\lambda}(\mathcal{L} F) \mathrm{d} s .
$$

Since we have seen in the previous section that this equation characterized $\mu^{*}$, we deduce that $\mu_{t}^{\lambda}=\mu_{t}^{*}$ for $t \in[0,1]$. Taking $\lambda=t_{0}^{-1}$ for $t_{0} \in[0,1]$, we deduce $\mu_{1}\left(F\left({\sqrt{t_{0}}}^{-1} X\right)\right)=$ $\mu_{t_{0}}(F)$ or equivalently (5.8). 
In the setting of Examples (2.4) (a) or (b) we can more precisely identify the limit law of the spectral measure of $\left(X_{N}(t), t \in[0,1]\right)$. In fact, let

$$
\psi(x, y)=\lim _{N \rightarrow \infty} \psi_{N}([N x],[N y])
$$

and denote by $K$ the operator in $L^{2}([0,1])$ with kernel $\psi$. Then

Lemma (5.10). - Let $k:[0,1] \times \mathbb{C} \backslash \mathbb{R} \rightarrow \mathbb{C}$ be the unique analytic solution of the nonlinear equation

$$
k(x, z)=(z-K(k(., z))(x))^{-1}
$$

so that $z k(x, z)$ goes to one as $|z|$ goes to infinity for any $x \in[0,1]$. Then, for any $\phi \in \mathcal{C}_{b}([0,1], \mathbb{R})$, any $t \in[0,1]$,

$$
\mu_{t}^{*}\left(\Delta(\phi)(z-X)^{-1}\right)=\frac{1}{\sqrt{t}} \int_{0}^{1} \phi(x) k\left(x, \frac{z}{\sqrt{t}}\right) \mathrm{d} x .
$$

This result is analogous to that found in [23] and [11].

Proof. - Note first that by (5.1),

$$
\begin{aligned}
& \partial_{t} \mu_{t}^{*}\left(\Delta(\phi)(z-X)^{-1}\right) \\
& \quad=-\frac{1}{2} \partial_{z} \int \mathrm{d} p(\tau) \mu_{t}^{*}\left(\Delta\left(\phi \sigma_{\tau}\right)(z-X)^{-1}\right) \mu_{t}^{*}\left(\Delta\left(\sigma_{\tau}\right)(z-X)^{-1}\right) .
\end{aligned}
$$

Further, according to Lemma (5.7),

$$
\begin{aligned}
\mu_{t}^{*}\left(\Delta(\phi)(z-X)^{-1}\right) & =\mu_{1}^{*}\left(\Delta(\phi)(z-\sqrt{t} X)^{-1}\right) \\
& =\sqrt{t}^{-1} \mu_{1}^{*}\left(\Delta(\phi)\left(\sqrt{t}^{-1} z-X\right)^{-1}\right)
\end{aligned}
$$

so that we get by derivation over $t \in[0,1]$,

$$
\partial_{t} \mu_{t}^{*}\left(\Delta(\phi)(z-X)^{-1}\right)=-\frac{1}{2 t} \mu_{t}^{*}\left(\Delta(\phi)(z-X)^{-1}\right)-\frac{z}{2 t} \partial_{z} \mu_{t}^{*}\left(\Delta(\phi)(z-X)^{-1}\right) .
$$

(5.11) and (5.13) result with

$\partial_{z}\left(z \mu_{t}^{*}\left(\Delta(\phi)(z-X)^{-1}\right)\right)=t \partial_{z}\left(\int \mathrm{d} p(\tau) \mu_{t}^{*}\left(\Delta\left(\phi \sigma_{\tau}\right)(z-X)^{-1}\right) \mu_{t}^{*}\left(\Delta\left(\sigma_{\tau}\right)(z-X)^{-1}\right)\right)$.

Noting that $\lim _{|z| \rightarrow \infty} z \mu_{t}^{*}\left(\Delta(\phi)(z-X)^{-1}\right)=\delta_{0}(\Delta(\phi))$, we get by integration over $z$,

$$
\begin{aligned}
z \mu_{t}^{*}\left(\Delta(\phi)(z-X)^{-1}\right)= & \delta_{0}(\Delta(\phi))+t \int \mathrm{d} p(\tau) \mu_{t}^{*}\left(\Delta\left(\phi \sigma_{\tau}\right)(z-X)^{-1}\right) \\
& \times \mu_{t}^{*}\left(\Delta\left(\sigma_{\tau}\right)(z-X)^{-1}\right) .
\end{aligned}
$$

Now, observe that for any $t \in[0,1]$ and $z \in \mathbb{C} \backslash \mathbb{R}, \phi \rightarrow \mu_{t}^{*}\left(\Delta(\phi)(z-X)^{-1}\right)$ is a linear bounded map on $L^{2}([0,1])$ since, by Cauchy-Schwartz's inequality

$$
\left|\mu_{t}^{*}\left(\Delta(\phi)(z-X)^{-1}\right)\right| \leqslant|\Im(z)|^{-1} \delta_{0}\left(\Delta(\phi)^{2}\right)^{\frac{1}{2}}=|\Im(z)|^{-1}|\phi|_{L^{2}([0,1])} .
$$


Hence, Riesz's theorem shows that there exists $k_{t}(., z) \in L^{2}([0,1])$ such that for any $\phi \in L^{\infty}([0,1])$

$$
\mu_{t}^{*}\left(\Delta(\phi)(z-X)^{-1}\right)=\int_{0}^{1} \phi(x) k_{t}(x, z) \mathrm{d} x .
$$

We deduce from (5.12) that for almost all $x \in[0,1]$,

$$
k_{t}(x, z)=\sqrt{t}^{-1} k_{1}\left(x, \sqrt{t}^{-1} z\right)
$$

and from (5.14) that for any $\phi \in L^{2}([0,1])$,

$$
\int_{0}^{1} \mathrm{~d} x \phi(x)\left(z k_{1}(x, z)-k_{1}(x, z) K\left(k_{1}(., z)\right)(x)\right) \mathrm{d} x=\int_{0}^{1} \phi(x) \mathrm{d} x
$$

so that for almost all $x \in[0,1]$,

$$
k_{1}(x, z)=(z-K(k(., z))(x) k(x, z))^{-1} .
$$

(5.15) and (5.17) give Lemma (5.10).

\subsection{Law of large numbers}

As a direct consequence of Lemma (5.2),

Lemma (5.18). - For any $F \in \overline{\mathcal{F}}_{\mathbb{C}}(X, \mathcal{D})$, $\left(\operatorname{tr}_{N}\left(F\left(X_{N}(t)\right)\right)\right)_{t \in[0,1]}$ converges almost surely towards $\left(\mu_{t}^{*}(F)\right)_{t \in[0,1] \text {. }}$

We can also improve the law of large numbers stated in Lemma (5.18) by enlarging the set of test functions. Indeed, denoting $P_{\mathbb{C}}(X, \mathcal{D})$ the set of non-commutative polynomial functions of $X$ and elements of $\mathcal{D}$, we have

Lemma (5.19). - For any polynomial function $P$ of $P_{\mathbb{C}}(X, \mathcal{D}),\left(\hat{\mu}_{t}^{(N)}(P)\right)_{t \in[0,1]}$ converges in $L^{\infty-}(\mathbb{P})=\bigcap_{q \in \mathbb{N}} L^{q}(\mathbb{P})$ towards $\left(\mu_{t}^{*}(P)\right)_{t \in[0,1]}$. In other words, for any $q \in \mathbb{N}$,

$$
\lim _{N \rightarrow \infty} \sup _{t \in[0,1]} \mathbb{E}\left[\left|\hat{\mu}_{t}^{(N)}(P)-\mu_{t}^{*}(P)\right|^{q}\right]=0 .
$$

Proof. - We can of course restrict ourselves to

$$
P(X)=\prod_{1 \leqslant i \leqslant n}^{\rightarrow} X \Delta_{i}(X)
$$

for $\left(\Delta_{i}\right)_{1 \leqslant i \leqslant n} \in \mathcal{D}$ since the $\Delta_{i}$ 's can be identically equal to identity. Set, for $\varepsilon>0$,

$$
P_{\varepsilon}(X)=(i)^{n} \prod_{1 \leqslant i \leqslant n} \frac{X}{i+\varepsilon X} \Delta_{i}(X) \in F_{\mathbb{C}}(X, \mathcal{D}) .
$$


Then, for any $t \in[0,1]$,

$$
\left|\operatorname{tr}_{N}\left(P_{\varepsilon}\left(X_{N}(t)\right)\right)-\operatorname{tr}_{N}\left(P\left(X_{N}(t)\right)\right)\right| \leqslant \varepsilon n\left(\prod_{1 \leqslant i \leqslant n}\left\|\Delta_{i}\right\|_{\infty}\right) \operatorname{tr}_{N}\left[X_{N}(t)^{2 n}\right]^{\frac{1}{2}} .
$$

Note that

$$
\mathbb{E}\left[\operatorname{tr}_{N}\left[X_{N}(t)^{2 n}\right]\right]=\frac{1}{N} \sum_{\gamma \in \Gamma} \mathbb{E}\left[\prod_{b_{i} \in \gamma} X_{b_{i}}^{N}(t)\right]
$$

with $\Gamma$ the set of connected bonds $b=(i, j)$ in $\{1, \ldots, N\}^{2}$ of length $2 n$ so that $b_{i+1} \simeq b_{i}$ if

$$
b \simeq b^{\prime} \quad \leftrightarrow \quad b=(i, j), b^{\prime}=(j, k), i, j, k \in\{1, \ldots, N\}^{3}
$$

and $b_{2 n} \simeq b_{1}$. In the right-hand side of (5.21), only the contours $\gamma$ so that if $b=(i, j) \in$ $\gamma, b^{*}=(j, i) \in \gamma$ with equal degree contribute, so that

$$
\mathbb{E}\left[\operatorname{tr}_{N}\left[X_{N}(t)^{2 n}\right]\right]=\frac{1}{N} \sum_{\gamma \in \Gamma} \mathbb{E}\left[\prod_{b, b^{*} \in \gamma} X_{b}^{N}(t) X_{b^{*}}^{N}(t)\right] .
$$

But, with $b=(i, j)$,

$$
X_{b}^{N}(t) X_{b^{*}}^{N}(t)=\psi_{N}(i, j) H_{b}^{N}(t) H_{b^{*}}^{N}(t)
$$

so that, since $H_{b}^{N}(t) H_{b^{*}}^{N}(t) \geqslant 0$ for any $b \in\{1, \ldots, N\}$, we deduce from (5.22) that

$$
\mathbb{E}\left[\operatorname{tr}_{N}\left[X_{N}(t)^{2 n}\right]\right] \leqslant T^{n} \mathbb{E}\left[\operatorname{tr}_{N}\left[H_{N}(t)^{2 n}\right]\right] .
$$

It is well known (see, for instance, [22], Theorem 2) that for any $n \in \mathbb{N}$,

$$
\sup _{N \in \mathbb{N}} \sup _{t \in[0,1]} \mathbb{E}\left[\operatorname{tr}_{N}\left[H_{N}(t)^{2 n}\right]\right]<\infty
$$

so that (5.23) results with, for any $n \in \mathbb{N}$,

$$
\sup _{N \in \mathbb{N}} \sup _{t \in[0,1]} \mathbb{E}\left[\operatorname{tr}_{N}\left[X_{N}(t)^{2 n}\right]\right]<\infty .
$$

With (5.20), we find, for any $q \in \mathbb{N}$, a finite constant $C(P, q)$ so that

$$
\sup _{N \in \mathbb{N}} \sup _{t \in[0,1]} \mathbb{E}\left[\left|\operatorname{tr}_{N}\left(P_{\varepsilon}\left(X_{N}(t)\right)\right)-\operatorname{tr}_{N}\left(P\left(X_{N}(t)\right)\right)\right|^{q}\right] \leqslant C(P, q) \varepsilon^{q} .
$$

Recalling by the previous proof that

$$
\lim _{\varepsilon \downarrow 0} \sup _{t \in[0,1]}\left|\mu_{t}^{*}\left(P_{\varepsilon}\right)-\mu_{t}^{*}(P)\right|=0
$$


and by Lemma (5.18) for any $\varepsilon>0$ (since $P_{\varepsilon}$ is uniformly bounded so that dominated convergence theorem applies)

$$
\lim _{N \rightarrow \infty} \mathbb{E}\left[\sup _{t \in[0,1]}\left|\operatorname{tr}_{N}\left(P_{\varepsilon}\left(X_{N}(t)\right)\right)-\mu_{t}^{*}\left(P_{\varepsilon}\left(X_{N}(t)\right)\right)\right|^{q}\right]=0
$$

we deduce from (5.25) that for any $q \in \mathbb{N}$,

$$
\lim _{N \rightarrow \infty} \sup _{t \in[0,1]} \mathbb{E}\left[\left|\operatorname{tr}_{N}\left(P\left(X_{N}(t)\right)\right)-\mu_{t}^{*}\left(P\left(X_{N}(t)\right)\right)\right|^{q}\right]=0 .
$$

\section{Central limit theorem}

In this section, we shall assume that $\mathcal{D}$ satisfies additionally the hypotheses $(\mathrm{H} 1)$ and $(\mathrm{H} 2)$ of Section 2. We shall then study the fluctuations of $\hat{\mu}_{t}^{(N)}(P)$ for $t \in$ $[0,1]$ and $P \in P_{\mathbb{C}}(X, \mathcal{D})$. This is equivalent, by the scaling property to study the fluctuations of $\left\{\hat{\mu}_{1}^{(N)}(P), P \in P_{\mathbb{C}}(X, \mathcal{D})\right\}$. This result is slightly less powerful than what T. Cabanal-Duvillard proved in [9], where fluctuations on path space for noncommutative functionals of independent Hermitian Brownian motions were obtained. However, to our point of view, the exhibited covariance functions are simpler here and the generalization to path space somehow not so much motivated.

To describe the mean and the covariance of the limiting Gaussian variables, we shall introduce the following operators on $P_{\mathbb{C}}(X, \mathcal{D})$.

We first let $X . \partial_{X}$ be the differential operator in $P_{\mathbb{C}}(X, \mathcal{D})$ given by

$$
X . \partial_{X} P=D_{X} P \sharp X=\left.\partial_{t} P(t X)\right|_{t=1} .
$$

As a counter part, we let $\mathcal{I}$ be given by

$$
\mathcal{I}(P)(X)=\int_{0}^{1} P(\sqrt{u} X) \mathrm{d} u .
$$

We define second order operators by

$$
L^{M}=\left(\mu_{1}^{*} \circ \mathcal{I} \otimes I+I \otimes \mu_{1}^{*} \circ \mathcal{I}\right) \circ \mathcal{L}
$$

and

$$
\Xi=X . \partial_{X}-2 \mu_{1}^{*} \otimes I \circ \mathbb{L} .
$$

Let $P_{\mathbb{R}}(X, \mathcal{D})$ be the subset of $P_{\mathbb{C}}(X, \mathcal{D})$ of Hermitian-valued polynomial functions. We recall that according to Lemma (5.3), any $P \in P_{\mathbb{R}}(X, \mathcal{D})$ belongs to $L^{2}\left(\mu_{1}^{*}\right)$. We shall prove that

THEOREM (6.1). -

(1) $I+\Xi$, as an operator from $P_{\mathbb{R}}(X, \mathcal{D})$ into $P_{\mathbb{R}}(X, \mathcal{D})$, is symmetric and invertible. Further, $(I+\Xi)^{-1}$ is a non-negative operator from $P_{\mathbb{R}}(X, \mathcal{D})$ into $P_{\mathbb{R}}(X, \mathcal{D})$, e.g., for 
any $P \in P_{\mathbb{R}}(X, \mathcal{D})$,

$$
\left\langle P,(I+\Xi)^{-1} P\right\rangle_{L^{2}\left(\mu_{1}^{*}\right)} \geqslant 0 .
$$

Further, if we set $(A \otimes B)^{t}=B \otimes A$ and for $Q \in P_{\mathbb{R}}(X, \mathcal{D}), D_{X} Q^{t}(X)=\left(D_{X} Q(X)\right)^{t}$ for all $X \in \mathcal{H}$, we have the more explicit formula for all $P, Q \in P_{\mathbb{R}}(X, \mathcal{D})$,

$$
\mu_{1}^{*}(P \Xi Q)=\int \mu_{1}^{*} \circ m_{\tau} \otimes \mu_{1}^{*} \circ m_{\tau}\left(D_{X} Q \times\left(D_{X} P\right)^{t}\right) \mathrm{d} p(\tau) .
$$

(2) If $(\mathrm{H} 1)$ and $(\mathrm{H} 2)$ are verified, for any $P \in P_{\mathbb{R}}(X, \mathcal{D}), N\left(\hat{\mu}_{1}^{(N)}(P)-\mu_{1}^{*}(P)\right)$ converges in law towards a Gaussian variable with covariance

$$
C(P)=\int \mu_{1}^{*}\left(m_{\tau}\left(\mathcal{D}_{X} P\right)(I+\Xi)^{-1} m_{\tau}\left(\mathcal{D}_{X} P\right)\right) \mathrm{d} p(\tau)
$$

and mean

$$
M(P)=c_{0}\left(e^{L^{M}} P(0)\right) .
$$

Before going any further, let us detail the above result in the classical Wigner's case.

Remark (6.2). - In the Wigner's case where $\Delta_{\tau} \equiv 1$ and $P$ is a polynomial function of $X$ only, note that we find the result originally due to K. Johansson [18] and in this form in [9]. Note first that in this case $c_{0} \equiv 0$ and the asymptotic Gaussian law is centered. Moreover, $\mu_{1}^{*}$ is the semicircle law $\pi^{-1} \sqrt{4-x^{2}} \mathrm{~d} x$ and $\mathbb{L}$ can be seen as the operator from $P[X]$ into $P[X, Y]$ given by

$$
\mathbb{L} f(x, y)=(y-x)^{-1}\left(f^{\prime}(y)-\frac{f(y)-f(x)}{y-x}\right) .
$$

But, if $P V$ denotes the principal value, the Hilbert transform

$$
H\left(\mu_{1}^{*}\right)(y)=P V \int(y-x)^{-1} \mathrm{~d} \mu_{1}^{*}(x)
$$

is well known to be equal to $H\left(\mu_{1}^{*}\right)(y)=2^{-1} y$ on the support $\Lambda_{1}^{*}$ of $\mu_{1}^{*}$. Thus, we obtain on $\Lambda_{1}^{*}$ that, for any $f \in \mathcal{C}_{b}^{1}(\mathbb{R})$,

$$
\begin{aligned}
\Xi(f)(x) & =x f^{\prime}(x)-2 \int \mathrm{d} \mu_{1}^{*}(y)(x-y)^{-1}\left(f^{\prime}(x)-\frac{f(y)-f(x)}{y-x}\right) \\
& =2 P V \int \mathrm{d} \mu_{1}^{*}(y) \frac{f(x)-f(y)}{(y-x)^{2}} \\
& =-f(x)-2 P V \int \frac{f(y)}{(x-y)^{2}} \mathrm{~d} \mu_{1}^{*}(y) .
\end{aligned}
$$

In the last line, we used $P V \int(y-x)^{-2} \mathrm{~d} \mu_{1}^{*}(x)=-2^{-1}$ which can be obtained by formal derivation from the definition of the Hilbert transform of the semi-circular law. It can look at first false because it states that the integral of a non-negative quantity is negative, 
but one should be careful that we have to take the principal value and actually justify these equalities by going back to the definition of principal values.

From the second formula in (6.3) it is clear that $\Xi$ is a symmetric non-negative operator in $L^{2}\left(\mu_{1}^{*}\right)$ with

$$
\mu_{1}^{*}(f \Xi g)=\int \mathrm{d} \mu_{1}^{*}(x) \mathrm{d} \mu_{1}^{*}(y)\left(\frac{f(x)-f(y)}{x-y}\right)\left(\frac{g(x)-g(y)}{x-y}\right)
$$

giving the identification of $\Xi$ of Theorem (6.1) (1) since $D_{X} f$ can be seen as the symmetric function of two variables

$$
D_{X} f(x, y)=\frac{f(x)-f(y)}{x-y} .
$$

Further, from the last formula in (6.3), we obtain that

$$
(I+\Xi)(f)(x)=-2 P V \int \frac{f(y)}{(x-y)^{2}} \mathrm{~d} \mu_{1}^{*}(y)
$$

so that if we denote by $K$ the symmetric operator in $L^{2}\left(\mu_{1}^{*}\right)$ given by

$$
K(f)=\int \log |x-y|^{-1} f(y) \mathrm{d} \mu_{1}^{*}(y),
$$

we find for $x \in \Lambda_{1}^{*}$,

$$
(I+\Xi)(f)(x)=-2 \partial_{x} K\left(\left(p_{1}^{*} f\right)^{\prime} / p_{1}^{*}\right)(x)
$$

with $\mu_{1}^{*}(\mathrm{~d} x)=p_{1}^{*}(x) \mathrm{d} x$. Observe that $\left(p_{1}^{*} f\right)^{\prime} / p_{1}^{*}=\partial_{x}^{*} f$ with $\partial_{x}^{*}$ the dual of the derivative $\partial_{x}$ in $L^{2}\left(\mu_{1}^{*}\right)$. Hence, $(I+\Xi)$ is a definite positive symmetric operator in $L^{2}\left(\mu_{1}^{*}\right)$ and, for any continuously differentiable function $f$,

$$
C(P)=\mu_{1}^{*}\left(P^{\prime}(I+\Xi)^{-1} P^{\prime}\right)=\frac{1}{2} \mu_{1}^{*}\left(P K^{-1} P\right) .
$$

More generally, we can consider the fluctuations of the trace of polynomial functions of $X+\Delta$, with $X$ a Gaussian Wigner matrix as above and $\Delta=\Delta(\phi)$ a diagonal matrix satisfying the hypotheses of Example (2.9). We set $\mu=m_{\Delta(\phi)}$ and choose $\mathcal{D}=\{\Delta(\phi)\}$. Then, it is well known that $\mu_{1}^{*}$ is the joint law of $X$ with semi-circular law $\sigma$ and $D$ with law $\mu, D$ free from $X$. We set $A$ to be the algebra generated by $X+D$ and $\nu^{*} \in \mathcal{P}(\mathbb{R})$, $\nu^{*}=\left.\mu_{1}^{*}\right|_{A}$, the free convolution of $\mu$ and $\sigma$. In this case, observe that if $f, g$ are two polynomial functions of $X+D$,

$$
\mu_{1}^{*}\left(f X . \partial_{X} g\right)=\mu_{1}^{*}\left(f E[X \mid X+D] . \partial_{X} g\right)=v^{*}\left(f E[X \mid X+D] . \partial_{X} g\right) .
$$

Now, it is well known by Voiculescu (see [26], Corollary 3.9) that

$$
E[X \mid X+D](x)=P V \int(x-y)^{-1} \mathrm{~d} \nu^{*} .
$$


Thus, we can proceed as above to see that, for any $f, g \in A$,

$$
\mu_{1}^{*}(f \Xi g)=\int \mathrm{d} \nu^{*}(x) \mathrm{d} \nu^{*}(y)\left(\frac{f(x)-f(y)}{x-y}\right)\left(\frac{g(x)-g(y)}{x-y}\right)
$$

yielding again Theorem (6.1) (1). However, becaus $\Xi A \not \subset A, C(P)=\mu_{1}^{*}\left(P^{\prime}(1+\right.$ $\left.\Xi)^{-1} P^{\prime}\right) \neq \frac{1}{2} v^{*}\left(P K^{-1} P\right)$ with $K f(x)=\int \log |x-y|^{-1} f(y) \mathrm{d} v^{*}(y)$, in general.

The proof of Theorem (6.1) follows two steps; we first show that $N\left(\hat{\mu}_{1}^{(N)}(P)-\mu_{1}^{*}(P)\right)$ converges in law towards a centered Gaussian variable and then identifies the covariance of this Gaussian law.

\subsection{A central limit theorem}

Since Itô's calculus is again the basis of our approach, let us first quote that we can extend $\mathcal{L}$ and $\mathbb{L}$ to $P_{\mathbb{C}}(X, \mathcal{D})$ by saying that $D_{X}$ satisfies the non-commutative Leibnitz rule on $P_{\mathbb{C}}(X, \mathcal{D})$ and that for any $A \in \mathcal{H}$

$$
D_{X} X(A)=1 \otimes 1, \quad D_{X} \Delta=0 \otimes 0, \quad \forall \Delta \in \mathcal{D},
$$

We can extend naturally Lemma (3.5) by

Lemma (6.5). - For any $F \in \mathcal{C}^{1}\left([0,1], P_{\mathbb{R}}(X, \mathcal{D})\right)$, the statements of Lemma (3.5) are true.

Let us define, for $s \in[0,1]$, the differential operator $L_{s}$ on $P_{\mathbb{R}}(X, \mathcal{D})$ given by

$$
L_{s}=\left(\mu_{s}^{*} \otimes 1+1 \otimes \mu_{s}^{*}\right) \mathcal{L} .
$$

Note that $L_{s}$ reduces by one the degree of any polynomial function $P \in P_{\mathbb{R}}(X, \mathcal{D})$ as a function of $(X, \mathcal{D})$, and of two as a function of $X$. Hence, for any polynomial function $P \in P_{\mathbb{R}}(X, \mathcal{D})$, any $t \in[0,1]$, we can define

$$
P_{t}(X)=\mathrm{e}^{\int_{t}^{1} L_{s} \mathrm{~d} s} P(X) \in \mathcal{C}^{1}\left([0,1], P_{\mathbb{R}}(X, \mathcal{D})\right)
$$

as the unique solution of the differential equation

$$
\partial_{t} P_{t}(X)=-L_{t} P_{t}(X), \quad P_{1}=P .
$$

We shall prove that

LeMMA (6.8). - Under hypotheses (H1) and $(\mathrm{H} 2)$, for any $P \in P_{\mathbb{R}}(X, \mathcal{D}), N\left(\hat{\mu}_{1}^{(N)}(P)\right.$ $\left.-\mu_{1}^{*}(P)\right)$ converges in law towards a Gaussian variable with covariance

$$
\tilde{C}(P)=\int_{0}^{1} \int \mu_{t}^{*}\left[m_{\tau}\left(\mathcal{D}_{X} P_{t}\right) m_{\tau}\left(\mathcal{D}_{X}^{*} P_{t}\right)\right] \mathrm{d} p(\tau) \mathrm{d} t
$$

and mean $M(P)=c\left(P_{0}(0)\right)$. 
In the next section we shall show that $\tilde{C}(P)$ coincides with $C(P)$ defined in Theorem (6.1). Note that, by definition of $L^{M}$, we already have $c\left(\mathrm{e}^{L^{M}}(P)(0)\right)=$ $c\left(P_{0}(0)\right)$.

Proof of Lemma (6.8). - Let us first notice that (5.1) implies that

$$
\partial_{t} \mu_{t}^{*}\left(P_{t}\right)=\mu_{t}^{*}\left(\partial_{t} P_{t}\right)+\mu_{t}^{*} \otimes \mu_{t}^{*}\left(\mathcal{L} P_{t}\right)=-\mu_{t}^{*} \otimes \mu_{t}^{*}\left(\mathcal{L} P_{t}\right)
$$

so that Lemma (6.5) gives

$$
\mathrm{d} N\left(\hat{\mu}_{t}^{(N)}-\mu_{t}^{*}\right)\left(P_{t}\right)=N\left(\hat{\mu}_{t}^{(N)}-\mu_{t}^{*}\right) \otimes\left(\hat{\mu}_{t}^{(N)}-\mu_{t}^{*}\right)\left(\mathcal{L} P_{t}\right) \mathrm{d} t+N \mathrm{~d} Q_{P}^{(N)}(t)
$$

with $\left(N Q_{P}^{(N)}(t)\right)_{t \in[0,1]}$ a real-valued martingale with bracket

$$
\left\langle N Q_{P}^{(N)}\right\rangle_{t}=\int_{0}^{t} \int_{\operatorname{tr}_{N}}\left[m_{\tau}\left(\mathcal{D}_{X} P_{s}\left(X_{N}(s)\right)\right) m_{\tau}\left(\mathcal{D}_{X}^{*} P_{S}\left(X_{N}(s)\right)\right)\right] \mathrm{d} p(\tau) \mathrm{d} s .
$$

To show that the first term in the r.h.s. of (6.9) goes to zero in $L^{\infty-}$ as $N$ goes to infinity, we shall prove by induction that

Lemma (6.11). - For any $n \in \mathbb{N}$, any $P_{1}, \ldots, P_{n} \in P_{\mathbb{C}}(X, \mathcal{D})$,

$$
\sup _{t \in[0,1]} \sup _{\tau_{1}, \ldots, \tau_{n} \in \Sigma} \sup _{N \in \mathbb{N}} \mathbb{E}\left[\left(N\left(\hat{\mu}_{t}^{(N)}-\mu_{t}^{*}\right)\left(\prod_{1 \leqslant i \leqslant n} \Delta_{\tau_{i}} P_{i}\right)\right)^{q}\right]<\infty .
$$

Proof. - Let $|P|$ be the degree of a polynomial function $P$ that is, if

$$
P(X)=\sum_{k=1}^{M} \beta_{k}\left(\prod_{1 \leqslant i \leqslant n_{k}} \Delta_{i}^{k} X\right) \Delta_{i}^{n_{k}+1}
$$

for some $n_{k} \in \mathbb{N}, \Delta_{i}^{k} \in \mathcal{D} \backslash\{0\}, \beta_{k} \in \mathbb{R}$,

$$
|P| \equiv \max _{k \in\{1, \ldots, M\}} n_{k} .
$$

We let $P_{\mathbb{C}}^{M}(X, \mathcal{D})$ be the polynomial functions with degree less or equal to $M$. For $P \in P_{\mathbb{C}}^{0}(X, \mathcal{D}), P \in \mathcal{D}$ and (6.12) is fulfilled under (H2). Let $M$ be an integer number. Assume now that (6.12) has been proved for any any choice of $n \in \mathbb{N}, n \leqslant M$, and any $P_{1}, \ldots, P_{n} \in P_{\mathbb{C}}(X, \mathcal{D})$ so that $\sum_{i=1}^{n}\left|P_{i}\right| \leqslant M$. Take $P_{1}, \ldots, P_{n} \in P_{\mathbb{C}}(X, \mathcal{D})$ so that

$$
P=P_{\underline{\tau}}=\prod_{1 \leqslant i \leqslant n}^{\rightarrow} \Delta_{\tau_{i}} P_{i}
$$

has degree $M+1$. By Lemma (6.5), we find that 


$$
\begin{aligned}
N\left(\hat{\mu}_{t}^{(N)}-\mu_{t}^{*}\right)(P)= & N\left(\hat{\mu}_{0}^{(N)}-\mu_{0}^{*}\right)(P)+\int_{0}^{t}\left(N\left(\hat{\mu}_{s}^{(N)}-\mu_{s}^{*}\right)\right) \otimes \hat{\mu}_{s}^{(N)}(\mathcal{L} P) \mathrm{d} s \\
& +\int_{0}^{t} \mu_{s}^{*} \otimes\left(N\left(\hat{\mu}_{s}^{(N)}-\mu_{s}^{*}\right)\right)(\mathcal{L} P) \mathrm{d} s+N Q_{P}^{(N)}(t)
\end{aligned}
$$

with a martingale $\left(N Q_{P}^{(N)}(u), 0 \leqslant u \leqslant 1\right)$ with bracket

$$
\left\langle N Q_{P}^{(N)}\right\rangle_{u}=\int_{0}^{u} \int \operatorname{tr}_{N}\left[m_{\tau}\left(\mathcal{D}_{X} P\left(X_{N}(s)\right)\right) m_{\tau}\left(\mathcal{D}_{X}^{*} P\left(X_{N}(s)\right)\right)\right] \mathrm{d} p(\tau) \mathrm{d} s .
$$

Therefore, by Jensen's inequality, for any $q \in 2 \mathbb{N}$, any $t \in[0,1]$, we obtain

$$
\begin{aligned}
\mathbb{E}\left[\left(N\left(\hat{\mu}_{t}^{(N)}-\mu_{t}^{*}\right)(P)\right)^{q}\right] \leqslant & 4^{q} \mathbb{E}\left[\left(N\left(\hat{\mu}_{0}^{(N)}-\mu_{0}^{*}\right)(P)\right)^{q}\right] \\
& +4^{q} \int_{0}^{t} \mathbb{E}\left[\left(N\left(\hat{\mu}_{s}^{(N)}-\mu_{s}^{*}\right) \otimes \hat{\mu}_{s}^{(N)}(\mathcal{L} P)\right)^{q}\right] \mathrm{d} s \\
& +4^{q} \int_{0}^{t} \mathbb{E}\left[\left(\mu_{s}^{*} \otimes\left(N\left(\hat{\mu}_{s}^{(N)}-\mu_{s}^{*}\right)(\mathcal{L} P)\right)^{q}\right)\right] \mathrm{d} s \\
& +4^{q} \mathbb{E}\left[\left(N Q_{P}^{(N)}(t)\right)^{q}\right] .
\end{aligned}
$$

Notice that since $P_{i}(0) \in \mathcal{D}$ for $i \in\{1, \ldots, n\},(\mathrm{H} 2)$ implies that

$$
\sup _{\tau_{1}, \ldots, \tau_{n} \in \Omega} \sup _{N \in \mathbb{N}} \mathbb{E}\left[\left(N\left(\hat{\mu}_{0}^{(N)}-\mu_{0}^{*}\right)\left(\prod_{1 \leqslant i \leqslant n} \Delta_{\tau_{i}} P_{i}(0)\right)\right)^{q}\right]<\infty
$$

for any $P_{1}, \ldots, P_{n} \in P_{\mathbb{R}}(X, \mathcal{D})$.

Moreover, observe that

(i) For any $P$ in $P_{\mathbb{C}}^{M}(X, \mathcal{D}), M \in \mathbb{N}, \mathcal{L} P \in P_{\mathbb{C}}^{M-1}(X, \mathcal{D}) \otimes P_{\mathbb{C}}^{M-1}(X, \mathcal{D})$.

(ii) From the uniform bound hypothesis (H0) on the operator norm of $\left(\Delta_{\tau}\right)_{\tau \in \Omega}$ and (2.2), we find that for any $P_{1}, \ldots, P_{n} \in P_{\mathbb{C}}(X, \mathcal{D})$, any $q \in 2 \mathbb{N}$,

$$
\sup _{\tau_{1}, \ldots, \tau_{n} \in \Omega} \sup _{t \in[0,1]} \sup _{N \in \mathbb{N}} \mathbb{E}\left[\left(\hat{\mu}_{t}^{(N)}\left(P_{\underline{\tau}}\right)\right)^{q}\right]<\infty
$$

From these two points and our induction hypothesis (with the uniform property with respect to the $\tau$ 's in $\Omega$ ), we infer that

$$
\sup _{\tau_{1}, \ldots, \tau_{n} \in \Omega} \sup _{N \in \mathbb{N}} \int_{0}^{1} \mathbb{E}\left[\left(N\left(\hat{\mu}_{s}^{(N)}-\mu_{s}^{*}\right) \otimes \hat{\mu}_{s}^{(N)}\left(\mathcal{L} P_{\underline{\tau}}\right)\right)^{q}\right]<\infty
$$


as well as

$$
\sup _{\tau_{1}, \ldots, \tau_{n} \in \Omega} \sup _{N \in \mathbb{N}} \int_{0}^{1} \mathbb{E}\left[\left(N\left(\hat{\mu}_{s}^{(N)}-\mu_{s}^{*}\right) \otimes \mu_{s}^{*}\left(\mathcal{L} P_{\underline{\tau}}\right)\right)^{q}\right]<\infty .
$$

(iii) The third term in (6.14) can be bounded by Burkholder-Davis-Gundy's inequality which asserts that there exists for any $q \in 2 \mathbb{N}$ a finite constant $c_{q}$ so that

$$
\begin{aligned}
& \mathbb{E}\left[\sup _{0 \leqslant s \leqslant t}\left(N Q_{P}^{(N)}(s)\right)^{q}\right] \leqslant c_{q} \mathbb{E}\left[\left\langle N Q_{P_{\underline{\tau}}}^{(N)}\right\rangle_{t}^{\frac{q}{2}}\right] \\
& \quad \leqslant c_{q} \iint_{0}^{t} \int \mathbb{E}\left[\operatorname{tr}_{N}\left[m_{\tau}\left(\mathcal{D}_{X} P_{\underline{\tau}}\left(X_{N}(s)\right)\right) m_{\tau}\left(\mathcal{D}_{X}^{*} P_{\underline{\tau}}\left(X_{N}(s)\right)\right)\right]^{\frac{q}{2}}\right] \mathrm{d} p(\tau) \mathrm{d} s,
\end{aligned}
$$

where we have used in the last line (6.13). By remark (ii) above, we deduce

$$
\sup _{s \in[0,1]} \sup _{\tau_{1}, \ldots, \tau_{n} \in \Omega} \sup _{N \in \mathbb{N}} \mathbb{E}\left[\operatorname{tr}_{N}\left[m_{\tau}\left(\mathcal{D}_{X} P_{\underline{\tau}}\left(X_{N}(s)\right)\right) m_{\tau}\left(\mathcal{D}_{X}^{*} P_{\underline{\tau}}\left(X_{N}(s)\right)\right)\right]^{\frac{q}{2}}\right]<\infty
$$

and hence

$$
\sup _{\tau \in \Omega} \sup _{\tau_{1}, \ldots, \tau_{n} \in \Omega} \sup _{N \in \mathbb{N}} \mathbb{E}\left[\sup _{0 \leqslant s \leqslant t}\left(N Q_{P_{\underline{\tau}}}^{(N)}(s)\right)^{q}\right]<\infty
$$

Plugging (6.15), (6.17), (6.18) into (6.14) bound $\mathbb{E}\left[\left(N\left(\hat{\mu}_{t}^{(N)}-\mu_{t}^{*}\right)\left(\Delta_{\tau} P_{\tau}\right)\right)^{q}\right]$ uniformly in $t \in[0,1], \tau_{1}, \ldots, \tau_{n} \in \Omega$ and $N \in \mathbb{N}$ and thus completes the proof of the lemma.

We can now finish the proof of Lemma (6.8). Following (6.9), for any $P \in P(X, \mathcal{D})$,

$$
N\left(\hat{\mu}_{1}^{(N)}-\mu_{1}^{*}\right)(P)=N\left(\hat{\mu}_{0}^{(N)}-\mu_{0}^{*}\right)\left(P_{0}\right)+R_{N}(P)+N Q_{P}^{(N)}(1),
$$

where $R_{N}(P)$ is some reminder term. Indeed, observe that $P_{s}$ is for any $s \in[0,1]$ a polynomial function with coefficients uniformly bounded in time according to Lemma (5.3). The same observation holds for $\mathcal{L} P_{s}$ which coefficients on the monomial basis of $P_{\mathbb{C}}(X, \mathcal{D}) \otimes P_{\mathbb{C}}(X, \mathcal{D})$ can be uniformly bounded in time. As a consequence, Lemma (6.11) implies that for any $q \in 2 \mathbb{N}$,

$$
\sup _{N \in \mathbb{N}} N^{q} \mathbb{E}\left[\left|R_{N}(P)\right|^{q}\right]<\infty
$$

In particular, $R_{N}(P)$ converges almost surely towards zero by Borel-Cantelli's lemma. Recall now that $P_{0}(0)$ belongs to $\mathcal{D}$ so that,

$$
\lim _{N \rightarrow \infty} N\left(\hat{\mu}_{0}^{(N)}-\mu_{0}^{*}\right)\left(P_{0}\right)=c\left(P_{0}(0)\right)
$$

Turning to the study of the last term in the r.h.s. of (6.19), recall that we have defined $\left(N Q_{P}^{(N)}(t), t \in[0,1]\right)$, as a martingal with bracket defined in (6.10). Again, by the above remarks on the structure of $P_{s}$ and Lemma (5.19), we see that $\left\langle N Q_{P}^{(N)}\right\rangle_{t}$, for $t \in[0,1]$, 
converges in $L^{\infty-}$ (and in particular in probability) towards

$$
\tilde{C}_{t}(P)=\int_{0}^{t} \int \mu_{s}^{*}\left[m_{\tau}\left(\mathcal{D}_{X} P_{s}\right) m_{\tau}\left(\mathcal{D}_{X}^{*} P_{s}\right)\right] \mathrm{d} p(\tau) \mathrm{d} s .
$$

Note that $\tilde{C}_{t}(P)$ is bounded as a consequence of Lemma (5.3). This classically implies that $N Q_{P}^{(N)}(1)$ converges in law towards a centered Gaussian process with covariance $\tilde{C}(P)$. Indeed, taking $\lambda \in \mathbb{R}$, we know that, $\left(N Q_{P}^{(N)}(t), t \in[0,1]\right)$ being a local martingale, $\left(\exp \left\{i \lambda N Q_{P}^{(N)}(t)\right\}, t \in[0,1]\right)$ is a semi-martingale and for $t \in[0,1]$,

$$
\begin{aligned}
E[ & \left.\exp \left\{i \lambda N Q_{P}^{(N)}(t)\right\}\right] \mathrm{e}^{\frac{\lambda^{2}}{2} \tilde{C}_{t}(P)} \\
=1-\frac{\lambda^{2}}{2} \int_{0}^{t} \int E[ & \exp \left\{i \lambda N Q_{P}^{(N)}(s)+\frac{\lambda^{2}}{2} \tilde{C}_{s}(P)\right\}\left(\left(\hat{\mu}_{s}^{(N)}-\mu_{s}^{*}\right)\right. \\
& \left.\left.\times\left[m_{\tau}\left(\mathcal{D}_{X} P_{s}\right) m_{\tau}\left(\mathcal{D}_{X}^{*} P_{s}\right)\right]\right)\right] \mathrm{d} p(\tau) \mathrm{d} s .
\end{aligned}
$$

By Lemma (5.19), the last term in the above right-hand side goes to zero as $N$ goes to infinity. Thus, for any $\lambda \in \mathbb{R}, \lim _{N \rightarrow \infty} E\left[\exp \left\{i \lambda N Q_{P}^{(N)}(1)\right\}\right]=\mathrm{e}^{-\frac{\lambda^{2}}{2} \tilde{C}_{1}(P)}$, that is $N Q_{P}^{(N)}(1)$ converges in law towards a centered Gaussian variable with covariance $\tilde{C}(P)=\tilde{C}_{1}(P)$. This result with (6.21) and (6.20) gives Lemma (6.8).

\subsection{Study of the covariance}

In this last section, we give a more explicit formula for the covariances driving the previous central limit theorems. The first step of which is to study the operator $\Xi$ introduced in Theorem (6.1).

\subsubsection{Study of some operators in $L^{2}\left(\mu_{1}^{*}\right)$}

We shall in this paragraph obtain the following identities.

LEMMA (6.22). -

(1) For any $P, Q \in P_{\mathbb{C}}(X, \mathcal{D})$,

$$
\mu_{1}^{*}\left(Q \mathcal{D}_{X} \circ\left(\mu_{1}^{*} \otimes I+I \otimes \mu_{1}^{*}\right) \circ \mathcal{L}(P)\right)=\mu_{1}^{*}\left(Q \mu_{1}^{*} \otimes I \circ \mathbb{L} \circ \mathcal{D}_{X}(P)\right) .
$$

(2) For any $P, Q \in P_{\mathbb{C}}(X, \mathcal{D})$,

$\mu_{1}^{*}\left(P\left(\mu_{1}^{*} \otimes I \circ \mathbb{L}-\frac{1}{2} X . \partial_{X}\right) Q\right)=-\frac{1}{2} \int \mu_{1}^{*} \circ m_{\tau} \otimes \mu_{1}^{*} \circ m_{\tau}\left(D_{X} Q \times\left(D_{X} P\right)^{t}\right) \mathrm{d} p(\tau)$.

(3) $\Xi=X . \partial_{X}-2 \mu_{1}^{*} \otimes I \circ \mathbb{L}$ is a symmetric operator from $P_{\mathbb{R}}(X, \mathcal{D})$ into $P_{\mathbb{R}}(X, \mathcal{D})$. $I+\Xi: P_{\mathbb{R}}(X, \mathcal{D}) \rightarrow P_{\mathbb{R}}(X, \mathcal{D})$ is invertible. Its inverse $(I+\Xi)^{-1}: P_{\mathbb{R}}(X, \mathcal{D}) \rightarrow$ $P_{\mathbb{R}}(X, \mathcal{D})$ is symmetric non-negative for the scalar product $\langle., .\rangle_{L^{2}\left(\mu_{1}^{*}\right)}$, e.g., for any polynomial functions $P, Q \in P_{\mathbb{R}}(X, \mathcal{D})$,

$$
\left\langle P,(I+\Xi)^{-1} Q\right\rangle_{L^{2}\left(\mu_{1}^{*}\right)}=\left\langle Q,(I+\Xi)^{-1} P\right\rangle_{L^{2}\left(\mu_{1}^{*}\right)}, \quad \text { and } \quad\left\langle P,(I+\Xi)^{-1} P\right\rangle_{L^{2}\left(\mu_{1}^{*}\right)} \geqslant 0 .
$$


Proof. - Unfortunately, we could not prove this lemma directly from Eq. (5.1) defining the minimum $\mu_{1}^{*}$. Instead, we shall go back to properties of the Hermitian Brownian motion and deduce it by taking the large $N$ limit.

To prove the first point, let us take $P \in P_{\mathbb{C}}(X, \mathcal{D})$, and consider the derivatives of $\operatorname{tr}_{N} \otimes$ $\operatorname{tr}_{N} \circ \mathcal{L}\left(P\left(X_{N}\right)\right)$ with respect to the entries of the self adjoint matrix $X_{N}=\left(x_{i j}\right)_{1 \leqslant i, j \leqslant N}$ with $x_{i j}=(1 / \sqrt{2 N}) \psi_{N}(i, j)^{1 / 2}\left(h_{i j}+\sqrt{-1} \tilde{h}_{i j}\right)$ when $i<j$. We first observe that for any $i, j \in\{1, \ldots, N\}$, with $\left(\Delta_{i j}\right)_{k l}=\delta_{k l=i j}$,

$$
\partial_{x_{i j}} \operatorname{tr}\left(P\left(X_{N}\right)\right)=\operatorname{tr}\left(D P\left(X_{N}\right) \sharp \Delta_{i j}\right)=\left(\mathcal{D}_{X} P\left(X_{N}\right)\right)_{j i} .
$$

Now, recall that from (3.9) and (3.11),

$$
\operatorname{tr}_{N} \otimes \operatorname{tr}_{N} \mathcal{L}(P)\left(X_{N}\right)=\frac{1}{2 N} \sum_{i, j=1}^{N} \psi_{N}(i, j) \partial_{h_{j i}} \partial_{h_{i j}} \operatorname{tr}_{N}(P)\left(X_{N}\right)
$$

implying with (6.24), that since $\partial_{x_{i j}}$ commutes with $\partial_{h_{k l}}$, for any $i, j \in\{1, \ldots, N\}$, any $P \in P_{\mathbb{C}}(X, \mathcal{D})$,

$$
\partial_{x_{i j}} \operatorname{tr}_{N} \otimes \operatorname{tr}_{N} \mathcal{L}(P)=\frac{1}{2 N} \sum_{k, l=1}^{N} \psi_{N}(k, l) \partial_{h_{k l}} \partial_{h_{l k}}\left(\mathcal{D}_{X} P\right)\left(X_{N}\right)_{j i} .
$$

Since $\mathcal{L} P \in P_{\mathbb{C}}(X, \mathcal{D}) \otimes P_{\mathbb{C}}(X, \mathcal{D}),(6.24)$ gives

$$
\partial_{x_{i j}} \operatorname{tr}_{N} \otimes \operatorname{tr}_{N} \mathcal{L}(P)=\left(\left(\mathcal{D}_{X} \otimes \operatorname{tr}_{N}+\operatorname{tr}_{N} \otimes \mathcal{D}_{X}\right)(\mathcal{L}(P))\right)_{j i} .
$$

Further, by (3.11),

$$
\frac{1}{2 N} \sum_{k, l=1}^{N} \psi_{N}(k, l) \partial_{h_{k l}} \partial_{h_{l k}}\left(\mathcal{D}_{X} P\right)\left(X_{N}\right)_{j i}=\left(\operatorname{tr}_{N} \otimes I \circ \mathbb{L}\left(\mathcal{D}_{X} P\right)\left(X_{N}\right)\right)_{j i}
$$

proving with (6.26) and (6.27) that

$$
\left(\mathcal{D}_{X} \otimes \operatorname{tr}_{N}+\operatorname{tr}_{N} \otimes \mathcal{D}_{X}\right)(\mathcal{L}(P))\left(X_{N}\right)=\operatorname{tr}_{N} \otimes I \circ \mathbb{L}\left(\mathcal{D}_{X} P\right)\left(X_{N}\right) .
$$

As a consequence, for any $Q \in P_{\mathbb{C}}(X, \mathcal{D})$, we obtain

$$
\begin{aligned}
& \mathbb{E}\left[\operatorname{tr}_{N}\left[Q\left(X_{N}(1)\right)\left(\mathcal{D}_{X} \otimes \operatorname{tr}_{N}+\operatorname{tr}_{N} \otimes \mathcal{D}_{X}\right)(\mathcal{L}(P))\left(X_{N}(1)\right)\right]\right] \\
& \quad=\mathbb{E}\left[\operatorname{tr}_{N}\left[Q\left(X_{N}(1)\right) \operatorname{tr}_{N} \otimes I \circ \mathbb{L}\left(\mathcal{D}_{X} P\right)\left(X_{N}(1)\right)\right]\right] .
\end{aligned}
$$

Hence, using the law of large numbers Theorem (5.19), we obtain at the large $N$ limit Lemma (6.22) (1).

To prove the second part of the lemma, we recall first that the Ornstein-Uhlenbeck process

$$
\mathrm{d} y_{t}=\frac{1}{\sqrt{2 N}} \mathrm{~d} \beta(t)-\frac{1}{2} y_{t} \mathrm{~d} t
$$


with initial distribution $\gamma_{N}$, the centered Gaussian law with covariance $(2 N)^{-1}$, is stationary. We let $X_{N}^{O U}$ be the matrix-valued process constructed as $X_{N}$ but with, instead of independent Brownian motions

$$
\left(\frac{1}{\sqrt{2 N}} \beta_{i, j}, \frac{1}{\sqrt{2 N}} \beta_{k, l}^{\prime}\right)_{1 \leqslant i<j \leqslant N}^{1 \leqslant k<l \leqslant N} \text { and }\left(\frac{1}{\sqrt{N}} \beta_{i, i}\right)_{1 \leqslant i \leqslant N},
$$

independent copies $\left(y_{i, j}, y_{k, l}^{\prime}\right)^{1 \leqslant k<i<j \leqslant N}$ and $\left(\sqrt{2} y_{i, i}\right)_{1 \leqslant i \leqslant N}$ of the Ornstein-Uhlenbeck process (6.28). Note that for any time $t \in[0,1], X_{N}^{O U}(t)$ has the same law that $X_{N}(1)$. Let $L_{N}$ be the infinitesimal generator of $\left(y_{i, j}, y_{k, l}^{\prime}\right)^{1 \leqslant k<i \leqslant j \leqslant N}$,

$$
L_{N}=\frac{1}{4 N} \sum_{i \leqslant j}\left(\left(1+1_{i=j}\right) \partial_{y_{i j}^{2}}+1_{i \neq j} \partial_{\left(y_{i j}^{\prime}\right)^{2}}\right)-\frac{1}{2} \sum_{i \leqslant j}\left(y_{i j} \partial_{y_{i j}}+1_{i \neq j} y_{i j}^{\prime} \partial_{y_{i j}^{\prime}}\right) .
$$

It is well known that $L_{N}$ is a symmetric operator in $L^{2}\left(\gamma_{N}^{\otimes N^{2}}\right)$ and that, for any $f, g: \mathbb{R}^{N^{2}} \rightarrow \mathbb{R}$

$$
\gamma_{N}^{\otimes N^{2}}\left(f\left(-L_{N}\right)(g)\right)=\frac{1}{4 N} \sum_{i \leqslant j} \gamma_{N}^{\otimes N^{2}}\left(\left(1+1_{i=j}\right) \partial_{y_{i j}} f \partial_{y_{i j}} g+1_{i \neq j} \partial_{y_{i j}^{\prime}} f \partial_{y_{i j}^{\prime}} g\right) .
$$

Now, one can check as in (3.11) that for any $P \in P_{\mathbb{C}}(X, \mathcal{D}), L_{N} P=\left(\operatorname{tr}_{N} \otimes I \mathbb{L}-\right.$ $\left.\frac{1}{2} X . \partial_{X}\right) P$. Hence, (6.29) implies that for any $P, Q \in P_{\mathbb{C}}(X, \mathcal{D})$,

$$
\begin{aligned}
\gamma_{N}^{\otimes N^{2}} & \left(\operatorname{tr}_{N}\left(Q\left(X_{N}\right)\left(\operatorname{tr}_{N} \otimes I \mathbb{L}-\frac{1}{2} X . \partial_{X}\right) P\left(X_{N}\right)\right)\right) \\
& =\gamma_{N}^{\otimes N^{2}}\left(\operatorname{tr}_{N}\left(Q\left(X_{N}\right) L_{N} P\left(X_{N}\right)\right)\right)=\gamma_{N}^{\otimes N^{2}}\left(\operatorname{tr}_{N}\left(P\left(X_{N}\right) L_{N} Q\left(X_{N}\right)\right)\right) \\
& =\gamma_{N}^{\otimes N^{2}}\left(\operatorname{tr}_{N}\left(P\left(X_{N}\right)\left(\operatorname{tr}_{N} \otimes I \mathbb{L}-\frac{1}{2} X . \partial_{X}\right) Q\left(X_{N}\right)\right)\right) .
\end{aligned}
$$

Thus, applying again Lemma (5.19) since $X_{N}$ has, under $\gamma_{N}^{\otimes N^{2}}$ the same law that $X_{N}(1)$, we find

$$
\mu_{1}^{*}\left(Q\left(\mu_{1}^{*} \otimes I \mathbb{L}-\frac{1}{2} X . \partial_{X}\right) P\right)=\mu_{1}^{*}\left(P\left(\mu_{1}^{*} \otimes I \mathbb{L}-\frac{1}{2} X . \partial_{X}\right) Q\right)
$$

that is the symmetry of the operator $\left(\mu_{1}^{*} \otimes I \mathbb{L}-\frac{1}{2} X . \partial_{X}\right)$ in $P_{\mathbb{C}}(X, \mathcal{D})$. We can also find another definition of this symmetric operator thanks again to (6.29) which gives

$$
\begin{aligned}
\gamma_{N}^{\otimes N^{2}} & \left(\operatorname{tr}_{N}\left(Q\left(X_{N}\right) L_{N} P\left(X_{N}\right)\right)\right) \\
= & \frac{-1}{4 N^{2}} \sum_{l, k=1}^{N} \sum_{i \leqslant j} \gamma_{N}^{\otimes N^{2}}\left(\left(1+1_{i=j}\right) \partial_{y_{i j}}\left(Q\left(X_{N}\right)\right)_{l k} \partial_{y_{i j}}\left(P\left(X_{N}\right)\right)_{k l}\right. \\
& \left.+1_{i \neq j} \partial_{y_{i j}^{\prime}}\left(Q\left(X_{N}\right)\right)_{l k} \partial_{y_{i j}^{\prime}}\left(P\left(X_{N}\right)\right)_{k l}\right) .
\end{aligned}
$$

Now observe that, if $\left(\Delta_{i j}\right)_{k l}=\delta_{i j=k l}\left(\psi_{N}(i, j)\right)^{\frac{1}{2}}$, 


$$
\begin{aligned}
& \partial_{y_{i j}}\left(Q\left(X_{N}\right)\right)_{l k}=\left(D_{X} Q \sharp\left(\Delta_{i j}+\Delta_{i j}^{*}\right)\right)_{l k} \quad \text { if } i<j, \\
& \partial_{y_{i i}}\left(Q\left(X_{N}\right)\right)_{l k}=\left(D_{X} Q \sharp \Delta_{i i}\right)_{l k}, \\
& \partial_{y_{i j}^{\prime}}\left(Q\left(X_{N}\right)\right)_{l k}=\left(D_{X} Q \sharp\left(\sqrt{-1} \Delta_{i j}-\sqrt{-1} \Delta_{i j}^{*}\right)\right)_{l k} \quad \text { if } i<j,
\end{aligned}
$$

yielding

$$
\begin{aligned}
& \gamma_{N}^{\otimes N^{2}}\left(\operatorname{tr}_{N}\left(Q\left(X_{N}\right) L_{N} P\left(X_{N}\right)\right)\right) \\
& =-\frac{1}{2 N^{2}} \sum_{l, k=1}^{N} \sum_{i, j=1}^{N} \psi_{N}(i, j) \gamma_{N}^{\otimes N^{2}}\left(\left(D_{X} Q \sharp \Delta_{i j}\right)_{l k}\left(D_{X} P \sharp \Delta_{j i}\right)_{k l}\right) \\
& =-\frac{1}{2 N^{2}} \int \gamma_{N}^{\otimes N^{2}}\left(\operatorname{tr}_{N} \circ m_{\tau} \otimes \operatorname{tr}_{N} \circ m_{\tau}\left(D_{X} Q\left(X_{N}\right) \times D_{X} P^{t}\left(X_{N}\right)\right)\right) \mathrm{d} p(\tau) .
\end{aligned}
$$

Now, we can again use Lemma (5.19) to take the limit $N \rightarrow \infty$ and conclude that

$$
\mu_{1}^{*}\left(Q\left(\mu_{1}^{*} \otimes I \mathbb{L}-\frac{1}{2} X . \partial_{X}\right) P\right)=-\frac{1}{2} \int \mu_{1}^{*} \circ m_{\tau} \otimes \mu_{1}^{*} \circ m_{\tau}\left(D_{X} Q \times\left(D_{X} P\right)^{*}\right) \mathrm{d} p(\tau)
$$

which achieves the proof of the lemma.

For the last point of the lemma, let us first recall that $\Xi\left(P_{\mathbb{C}}(X, \mathcal{D})\right) \subset P_{\mathbb{C}}(X, \mathcal{D})$. Further, if $P \in P_{\mathbb{R}}(X, \mathcal{D}),(\Xi(P)(X))^{*}=\Xi(P)(X)$ because

- $X . \partial_{X} P=\lim _{\varepsilon \downarrow 0} \varepsilon^{-1}\left(P^{(1+\varepsilon)^{2}}-P\right)=\lim _{\varepsilon \downarrow 0} \varepsilon^{-1}\left(P^{(1+\varepsilon)^{2}}-P\right)^{*}=\left(X . \partial_{X} P\right)^{*}$.

- Similarly, $\mathbb{L}(P)=(\mathbb{L}(P))^{*}$ if $(A \otimes B)^{*}=B^{*} \otimes A^{*}$ from which one sees that

$$
\left(\mu_{1}^{*} \otimes I \circ \mathbb{L}(P)(X)\right)^{*}=\mu_{1}^{*} \otimes I \circ \mathbb{L}(P)(X) .
$$

Moreover, if we define formally $(I+\Xi)^{-1} \equiv \sum_{n \geqslant 0}(-\Xi)^{n}$, then $(I+\Xi)^{-1}$ is well defined on $P_{\mathbb{C}}(X, \mathcal{D})$ since for any $P \in P_{\mathbb{C}}(X, \mathcal{D})$, for $n$ large enough, $\Xi^{n} P \equiv 0$. Further, it is not hard to check that for any $P \in P_{\mathbb{C}}(X, \mathcal{D}),(I+\Xi)(I+\Xi)^{-1} P=(I+\Xi)^{-1}(I+$ $\Xi) P=P$, implying that $I+\Xi$ is invertible with inverse $(I+\Xi)^{-1}: P_{\mathbb{R}}(X, \mathcal{D}) \rightarrow$ $P_{\mathbb{R}}(X, \mathcal{D})$. Clearly, the symmetry of $\Xi: P_{\mathbb{R}}(X, \mathcal{D}) \rightarrow P_{\mathbb{R}}(X, \mathcal{D})$ implies that of $(I+$ $\Xi)^{-1}$. Finally, for any polynomial function $P \in P_{\mathbb{R}}(X, \mathcal{D})$, if we let $Q=(I+\Xi)^{-1} P \in$ $P_{\mathbb{R}}(X, \mathcal{D})$,

$$
\left\langle P,(I+\Xi)^{-1} P\right\rangle_{L^{2}\left(\mu_{1}^{*}\right)}=\left\langle(I+\Xi)^{-1} Q, Q\right\rangle_{L^{2}\left(\mu_{1}^{*}\right)} \geqslant 0
$$

since by (2), $\langle\Xi Q, Q\rangle_{L^{2}\left(\mu_{1}^{*}\right)} \geqslant 0$ for any $Q \in P_{\mathbb{R}}(X, \mathcal{D})$. The proof of the lemma is complete.

\subsubsection{Identification of the covariance}

Hereafter, a polynomial function $Q \in P_{\mathbb{R}}(X, \mathcal{D})$ will be fixed and we shall denote by $Q_{s}(X)=\mathrm{e}^{\int_{s}^{1} L_{u} \mathrm{~d} u} Q$. Set, for any $s \in[0,1]$, any $\tau \in \Omega$,

$$
\Lambda(s, \tau)=\mu_{s}^{*}\left[m_{\tau}\left(\mathcal{D}_{X} Q_{s}\right) m_{\tau}\left(\mathcal{D}_{X}^{*} Q_{s}\right)\right] .
$$


Note first that by Lemma (5.7), for any $s \in[0,1]$, any $\tau \in \Sigma$,

$$
\Lambda(s, \tau)=\mu_{1}^{*}\left[m_{\tau}\left(\left(\mathcal{D}_{X} Q_{s}\right)(\sqrt{s} X)\right) m_{\tau}\left(\left(\mathcal{D}_{X}^{*} Q_{s}\right)(\sqrt{s} X)\right)\right] .
$$

Further, since $\mathcal{D}_{X}$ is a derivative, $\left(\mathcal{D}_{X} P^{s}\right)(X)=\sqrt{s}\left(\mathcal{D}_{X} P\right)^{s}(X)$. Thus, (6.31) reads

$$
\Lambda(s, \tau)=\frac{1}{s} \mu_{1}^{*}\left[m_{\tau}\left(\left(\mathcal{D}_{X} Q_{s}^{s}\right)\right) m_{\tau}\left(\left(\mathcal{D}_{X}^{*} Q_{s}^{s}\right)\right)\right] .
$$

Now, by definition of $X . \partial_{X}$,

$$
\partial_{s} Q_{s}^{s}(X)=\left(-L_{s}+\frac{1}{2 s} X . \partial_{X}\right) Q_{s}(\sqrt{s} X) .
$$

But, since $\mathcal{L}$ is a second order operator, for any $s \in[0,1], \mathcal{L}\left(P^{s}\right)=s(\mathcal{L}(P))^{s}$, we find $\left(L_{s} P\right)^{s}=s^{-1} L_{1}\left(P^{s}\right)$. Thus, we deduce from (6.33) that

$$
\partial_{s} Q_{s}^{s}(X)=s^{-1}\left(-L_{1}+\frac{1}{2} X . \partial_{X}\right)\left(Q_{s}^{s}\right)(X)
$$

so that, for any $s>0$,

$$
Q_{s}^{s}(X)=\mathrm{e}^{\log (s)\left(-L_{1}+\frac{1}{2} X . \partial_{X}\right)}(Q)(X) .
$$

Remark that we can compute the commutator of $X . \partial_{X}$ and $\mathcal{D}_{X}$ since

$$
\begin{aligned}
\mathcal{D}_{X} \circ X . \partial_{X} P & =\lim _{\varepsilon \rightarrow 0} \varepsilon^{-1} \mathcal{D}_{X}\left(P^{(1+\varepsilon)^{2}}-P\right) \\
& =\lim _{\varepsilon \rightarrow 0} \varepsilon^{-1}\left((1+\varepsilon)\left(\mathcal{D}_{X} P\right)^{(1+\varepsilon)^{2}}-\mathcal{D}_{X} P\right) \\
& =\left(X . \partial_{X} \circ \mathcal{D}_{X}+\mathcal{D}_{X}\right) P .
\end{aligned}
$$

Thus,

$$
\mathcal{D}_{X} \circ\left(-L_{1}+\frac{1}{2} X . \partial_{X}\right)=\left(-\tilde{L}_{1}+\frac{1}{2} X . \partial_{X}+\frac{1}{2} I\right) \circ \mathcal{D}_{X}
$$

with

$$
\tilde{L}_{1}=\frac{1}{2} \mathcal{D}_{X} \circ\left(\mu_{1}^{*} \otimes 1+1 \otimes \mu_{1}^{*}\right) \int \mathrm{d} p(\tau) m_{\tau} \otimes m_{\tau} D_{X}
$$

Now, as an operator on $P_{\mathbb{C}}(X, \mathcal{D})$, we observed in Lemma (6.22) (1) that

$$
\Xi=-2 \mu_{1}^{*} \otimes I \mathbb{L}+X . \partial_{X}=-2 \tilde{L}_{1}+X . \partial_{X} .
$$

Plugging (6.34), (6.35) and (6.36) in (6.32) yields, with the observation that $m_{\tau}$ commutes with $\Xi$,

$$
\Lambda(s, \tau)=\frac{1}{s} \mu_{1}^{*}\left[\mathrm{e}^{\frac{1}{2} \log (s)(I+\Xi)} m_{\tau}\left(\mathcal{D}_{X} Q\right) \mathrm{e}^{\frac{1}{2} \log (s)(I+\Xi)} m_{\tau}\left(\mathcal{D}_{X} Q\right)\right] .
$$


Hence, we find that, since $I+\Xi$ is symmetric definite positive,

$$
\begin{aligned}
\tilde{C}(Q) & =\int_{0}^{1} \int \Lambda(s, \tau) \mathrm{d} p(\tau) \mathrm{d} s \\
& =\int_{0}^{1} \frac{1}{s} \int \mu_{1}^{*}\left[\mathrm{e}^{\frac{1}{2} \log (s)(I+\Xi)} m_{\tau}\left(\mathcal{D}_{X} Q\right) \mathrm{e}^{\frac{1}{2} \log (s)(I+\Xi)} m_{\tau}\left(\mathcal{D}_{X} Q\right)\right] \mathrm{d} p(\tau) \mathrm{d} s \\
& =\int_{0}^{\infty} \int \mu_{1}^{*}\left[\mathrm{e}^{-u(I+\Xi)}\left(m_{\tau}\left(\mathcal{D}_{X} Q\right)\right) m_{\tau}\left(\mathcal{D}_{X} Q\right)\right] \mathrm{d} p(\tau) \mathrm{d} u \\
& =\int_{1} \mu_{1}^{*}\left(m_{\tau}\left(\mathcal{D}_{X} Q\right)(I+\Xi)^{-1}\left(m_{\tau}\left(\mathcal{D}_{X} Q\right)\right)\right) \mathrm{d} p(\tau)
\end{aligned}
$$

which is by definition $C(Q)$. Here, one can check that the last line agrees with our definition of $(I+\Xi)^{-1}=\sum_{n \geqslant 0}(-\Xi)^{n}$ by expending the exponential in $\Xi$ (yielding only a finite sum) and integrating the polynomial function in $u$.

\section{Acknowledgements}

I am very grateful to T. Cabanal-Duvillard who drew my attention over band matrices and D. Shlyakhtenko's work. I thank O. Zeitouni whose comments allowed me to improve this paper, and in particular to obtain applications to generalized sample covariance matrices. I also wish to thank D. Voiculescu, D. Shlyakhtenko, A. Dembo and P. Biane for useful conversations, while working on this paper. Finally, while struggling with the covariance operator $\Xi$, I was encouraged by D. Ioffe and S. Olla who I thank for their patience. I thank the referee for his encouragments.

\section{REFERENCES}

[1] L. Arnold, On the asymptotic distribution of eigenvalues of random matrices, J. Math. Anal. Appl. 20 (1967) 262-268.

[2] Z.D. Bai, Methodologies in spectral analysis of large dimensional random matrices: a review, Statistica Sinica 9 (1999) 611-661.

[3] G. Ben Arous, A. Guionnet, Large deviations for Wigner's law and Voiculescu's noncommutative entropy, Probab. Theory Related Fields 108 (4) (1997) 517-542.

[4] G. Ben Arous, O. Zeitouni, Large deviations from the circular law, ESAIM Probab. Statist. 2 (1998) 123-134.

[5] F.A. Berezin, Some remarks on the Wigner distribution, Teor. Mat. Fiz. 17 (1973) $1163-$ 1171.

[6] P. Biane, Free brownian motion, free stochastic calculus and random matrices, in: D. Voiculescu (Ed.), Free Probability Theory, Fields Institute Communication, Vol. 12, American Math. Soc., 1997, pp. 1-19.

[7] P. Biane, Calcul stochastique non-commutatif, in: Saint Flour 1993, Lect. Notes in Math., Vol. 1608, Springer, Berlin, 1995, pp. 1-96.

[8] A. Boutet De Monvel, A.M. Khorunzhy, Limit theorems for random matrices, Markov Proc. Related Fields 4 (1998) 175-197. 
[9] T. Cabanal-Duvillard, Fluctuations de la loi spectrale des grandes matrices aléatoires, Ann. Inst. H. Poincaré (2000), to appear.

[10] T. Cabanal-Duvillard, A. Guionnet, Large deviations upper bounds and non commutative entropies for some matrices ensembles, Annals of Probab. (2001), to appear.

[11] G. Casati, V. Girko, Wigner's semicircle law for band matrices, Random Operators Stochastic Equations 1 (1993) 279-286.

[12] A. Dembo, O. Zeitouni, Large Deviations Techniques and Applications, Jones and Bartlett Publishers, 1993.

[13] F. Hiai, D. Petz, Eigenvalues density of the Wishart matrix and large deviations, Infinite Dimensional Anal. Quantum Prob. 1 (1998) 633-646.

[14] A. Guionnet, O. Zeitouni, Concentration of the spectral measure for large matrices, Elec. Comm. Prob. 5 (2000) 14.

[15] A. Guionnet, O. Zeitouni, Large deviations asymptotics for spherical integrals, J. Funct. Anal., in press.

[16] A.M. Khorunzhy, B.A. Khoruzhenko, L.A. Pastur, Asymptotic properties of large random matrices with independent entries, J. Math. Phys. 37 (1996) 5033-5060.

[17] A.M. Khorunzhy, B.A. Khoruzhenko, L.A. Pastur, M.V. Shcherbina, The large $n$-limit in statistical mechanics and the spectral theory of disordered systems phase, Transition and Critical Phenomena 15 (1992) 73-239.

[18] K. Johansson, On fluctuations of eigenvalues of random Hermitian matrices, Duke Math. J. 91 (1998) 151-204.

[19] C. Kipnis, S. Olla, S.R.S. Varadhan, Hydrodynamics and large deviation for dimple exclusion processes, Comm. Pure Appl. Math. 42 (1989) 115-137.

[20] L.A. Pastur, V.A. Martchenko, The distribution of eigenvalues in certain sets of random matrices, Math. USSR-Sbornik 72 (1967) 507-536.

[21] W. Rudin, Real and Complex Analysis, 3rd edn., McGraw-Hill, 1986.

[22] Y. Sinai, A. Soshnikov, Central limit theorem for traces of large random symmetric matrices with independent elements, Bol. Soc. Brasil. Math. 29 (1998) 1-24.

[23] D. Shlyakhtenko, Random Gaussian band matrices and freeness with amalgation, Int. Math. Res. Not. 20 (1996) 1013-1025.

[24] D. Shlyakhtenko, Free Fisher information with respect to a completely positive map and cost of equivalence relations, Preprint (1999).

[25] D. Voiculescu, The analogues of entropy and Fisher's information measure in free probability theory, I, Commun. Math. Phys. 155 (1993) 71-92.

[26] D. Voiculescu, The analogues of entropy and Fisher's information measure in free probability theory, V: Noncommutative Hilbert transforms, Invent. Math. 132 (1) (1998) 189-227.

[27] K.W. Wachter, The strong limits of random matrix spectra for sample matrices of independent elements, Ann. Probab. 6 (1978) 1-18.

[28] E. Wigner, On the distribution of the roots of certain symmetric matrices, Ann. Math. 67 (1958) 325-327.

[29] J. Wishart, The generalized product moment distribution in samples from a normal multivariate population, Biometrika 20 A (1928) 32-52. 